\title{
Tenomodulin is essential for prevention of adipocyte accumulation and fibrovascular scar formation during early tendon healing
}

\author{
Dasheng Lin ${ }^{1,2}$, Paolo Alberton ${ }^{1}$, Manuel Delgado Caceres ${ }^{3}$, Elias Volkmer ${ }^{1,4}$, Matthias Schieker ${ }^{1,5}$ and Denitsa Docheva ${ }^{\star, 3}$
}

Tenomodulin $(T n m d)$ is the best-known mature marker for tendon and ligament lineage cells. It is important for tendon maturation, running performance and has key implications for the resident tendon stem/progenitor cells (TSPCs). However, its exact functions during the tendon repair process still remain elusive. Here, we established an Achilles tendon injury model in a Tnmd knockout $\left(\mathrm{Tnmd}^{-1-}\right)$ mouse line. Detailed analyses showed not only a very different scar organization with a clearly reduced cell proliferation and expression of certain tendon-related genes, but also increased cell apoptosis, adipocyte and blood vessel accumulation in the early phase of tendon healing compared with their wild-type (WT) littermates. In addition, $\mathrm{Tnmd}^{-1-}$ tendon scar tissue contained augmented matrix deposition of biglycan, cartilage oligomeric matrix protein (Comp) and fibronectin, altered macrophage profile and reduced numbers of CD146-positive cells. In vitro analysis revealed that $\mathrm{Tnmd}^{-1-}$ TSPCs exhibited significantly reduced migration and proliferation potential compared with that of WT TSPCs. Furthermore, $\mathrm{Tnmd}^{-1-}$ TSPCs had accelerated adipogenic differentiation accompanied with significantly increased peroxisome proliferator-activated receptor gamma (Ppary) and lipoprotein lipase (LpI) mRNA levels. Thus, our results demonstrate that Tnmd is required for prevention of adipocyte accumulation and fibrovascular scar formation during early tendon healing.

Cell Death and Disease (2017) 8, e3116; doi:10.1038/cddis.2017.510; published online 12 October 2017

Tendon injuries are some of the most common orthopedic problems accounting for substantial pain, disability, and economic burden. ${ }^{1}$ While many tendon injuries are acute, a very large number are chronic causing degenerative conditions. ${ }^{2}$ Repair in either case results in the formation of fibrovascular scar, fat deposition or heterotopic ossification that never attains the gross, histological, or mechanical characteristics of normal tendon. ${ }^{3-5}$ The precise mechanisms of matrix degeneration, tissue tearing, and the subsequent repair process remain poorly understood. ${ }^{1}$ Tenomodulin (Tnmd) is a member of a novel class protein family of type II transmembrane glycoproteins with a highly conserved cleavable C-terminal cysteine-rich domain. ${ }^{6,7}$ The Tnmd gene consists of seven exons localized on the $X$ chromosome and accounts for an $\sim 1.4 \mathrm{~kb}$ transcript and a predicted full-length protein consisting of 317 amino acids. $^{6,7}$ It is predominantly expressed in tendons and ligaments, but low levels of mRNA transcripts have also been identified in other tissues. ${ }^{6-9}$ Tnmd is the bestknown marker of the mature tendon and ligament lineage with a suggested dual role of its C-terminal domain, namely a pro-proliferative action with tendon/ligament cells and anti-angiogenic potential with vascular cells. ${ }^{9,10}$ Interestingly, loss of Tnmd expression in gene targeted mice $\left(\mathrm{Tnmd}^{-1}\right.$ ) abated tenocyte proliferation, led to reduced tenocyte density and to pathological thickening of collagen fibers in the tendon extracellular matrix (ECM) in vivo but caused no major changes in the tendon vasculature. ${ }^{11}$ In our recent study, we subjected $\mathrm{Tnmd}^{-/}$mice and their wild-type (WT) littermates to exhaustive running tests revealing significantly inferior running performance of the knockouts that further worsened with training. ${ }^{12}$ In vitro analysis of $\mathrm{Tnmd}^{-1}$ tendon stem/progenitor cells (TSPCs) showed significantly reduced self-renewal, and augmented senescence paralleled by upregulated p53 mRNA levels, which was confirmed in vivo by detecting an increased number of p53-positive tenocytes in Tnmo $^{-1-}$ Achilles tendons. ${ }^{13}$ In addition, overexpression of Tnmd in murine mesenchymal stem cells (MSCs) inhibited their commitment towards the adipogenic, chondrogenic and osteogenic lineages, whilst promoting their tenogenic differentiation. ${ }^{14}$ The above data motivated us to further examine the potential regulatory role of Tnmd gene in the early tendon healing stage when major cellular and ECM events take place, ${ }^{3}$ such as vascular and inflammatory cell invasion, intrinsic cell activation, migration and proliferation, and ECM deposition. Hence, the objective of this study was to investigate the functions of Tnmd in early tendon healing in vivo and in wound healing assays in vitro, including careful tissue phenotyping and specific molecular target analyses, using the $\mathrm{Tnmd}^{-/}$mouse strain.

\footnotetext{
${ }^{1}$ Experimental Surgery and Regenerative Medicine, Department of Surgery, Ludwig-Maximilians-University (LMU), Munich, Germany; ${ }^{2}$ Orthopaedic Center of People's Liberation Army, Xiamen University Affiliated Southeast Hospital, Zhangzhou, China; ${ }^{3}$ Experimental Trauma Surgery, Department of Trauma Surgery, University Regensburg Medical Centre, Regensburg, Germany; ${ }^{4}$ Department of Hand, Plastic and Aesthetic Surgery, LMU, Munich, Germany and ${ }^{5}$ Novartis Institutes for Biomedical Research (NIBR), Translational Medicine Musculoskeletal Disease, Basel, Switzerland

${ }^{*}$ Corresponding author: D Docheva, Experimental Trauma Surgery, Department of Trauma Surgery University Regensburg Medical Centre, Franz-Josef-Strauss-Allee 11, Regensburg, 93053 Germany. Tel: +49(0)9419431605; Fax: +49(0)9419431631; E-mail: denitsa.docheva@ukr.de

Received 10.3.17; revised 19.7.17; accepted 20.7.17; Edited by D Aberdam
} 
a
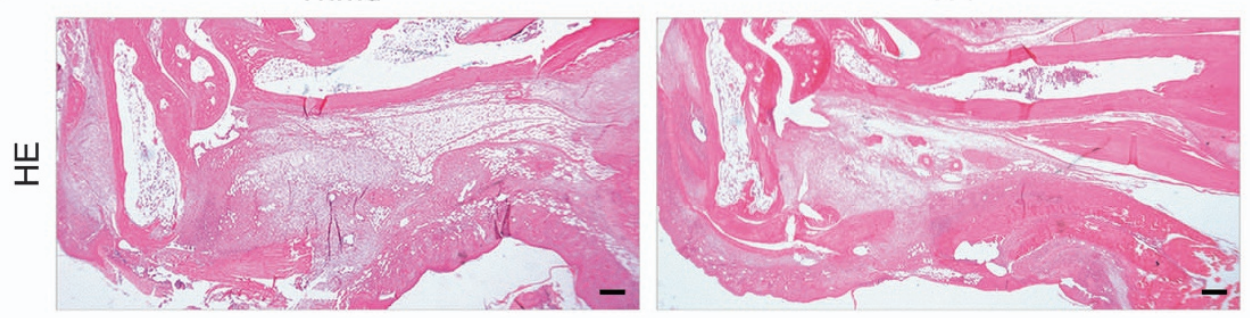

C

$\frac{1}{0}$

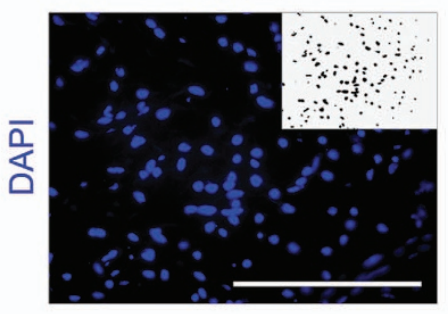

f

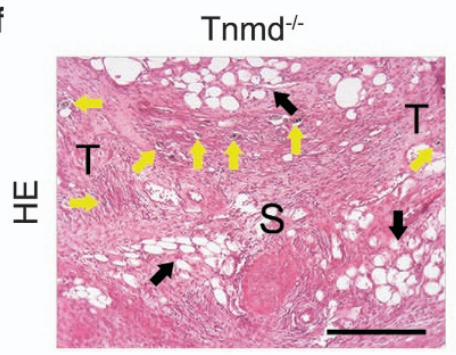

i
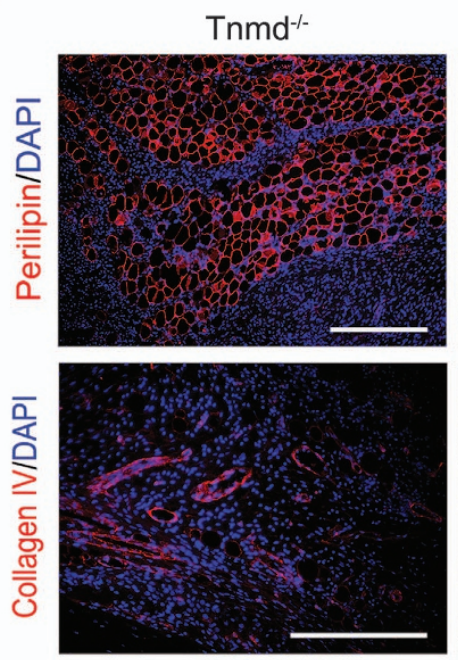

WT

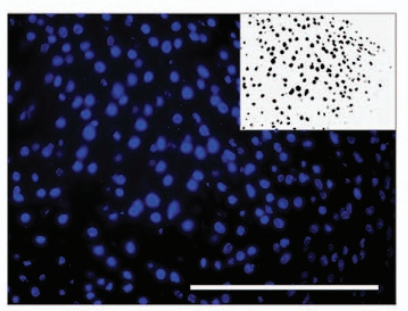

WT
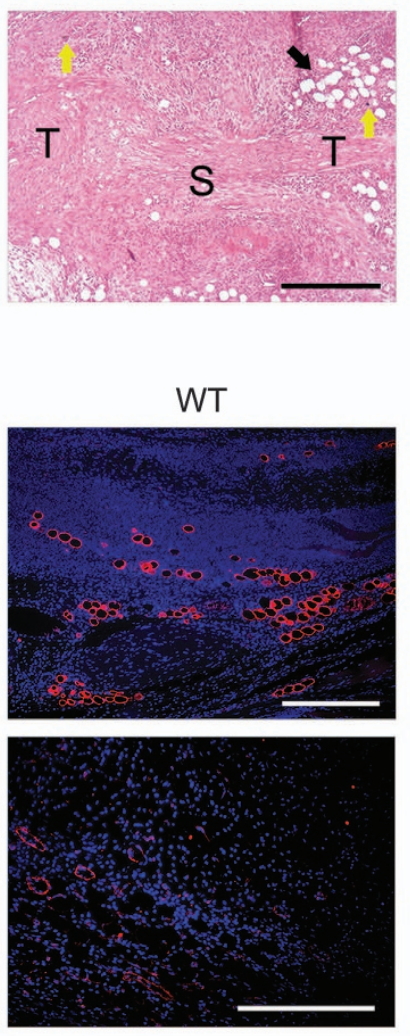

d

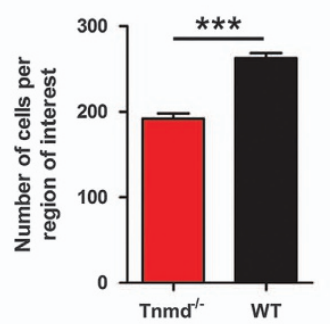

g

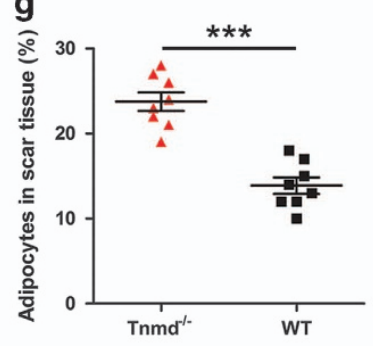

j

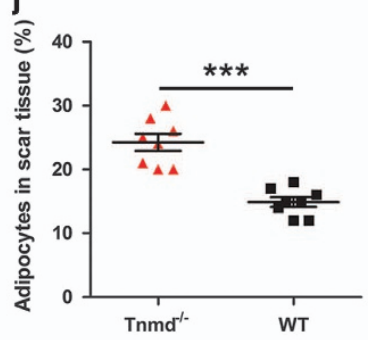

b

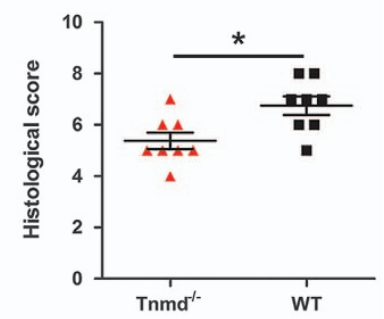

e

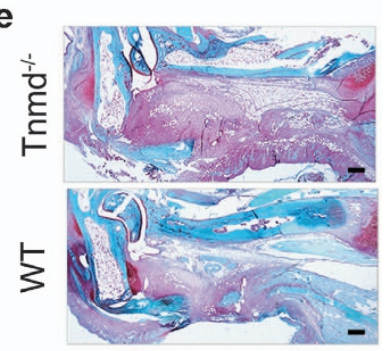

h

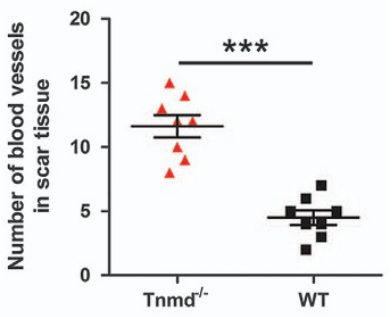

$\mathbf{k}$

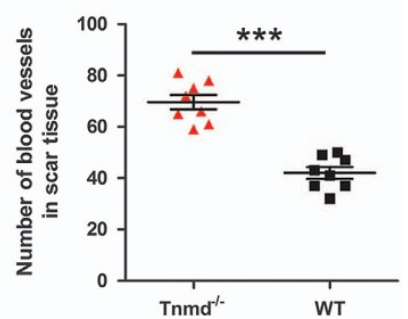

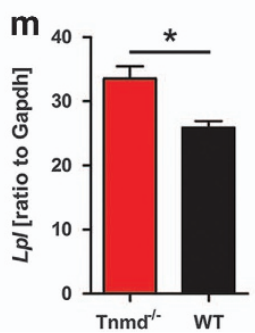

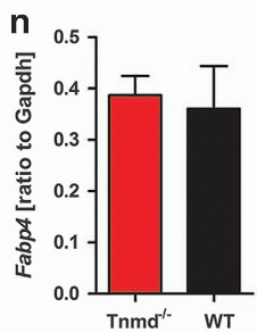

\section{Results}

$\mathrm{Tnmd}^{-/}$tendon scars have inferior gross appearance, histological scores and cell density paralleled with increased accumulation of adipocytes and vessels. To analyze Tnmd involvement during early tendon healing, we established a mouse model of full thickness Achilles tendon injury. ${ }^{15}$ We analyzed the mice eight days after surgical repair, a time point characterized by scar formation, vascular and inflammatory cell invasion, high cell migration and proliferation as well as robust ECM secretory activity. .,16 $^{2}$ Hematoxylin-eosin (HE) staining of sectioned tendons revealed a very different scar organization in $\mathrm{Tnmd}^{-1-}$ mice, as indicated by significantly inferior total histological scores ${ }^{17}$ (Supplementary Table 1) compared with their WT littermates 
Figure 1 Tnmd deficiency results in an inferior tendon repair process, lower cell density and increased adipocyte and vessel accumulation. (a) Low-magnification HE staining indicates a very different scar organization with clear adipocyte accumulation in $\mathrm{Tnmd}^{-/}$mice. (b) Evaluation of tendon healing using an established histological scoring system revealed that $\mathrm{Tnmd}^{-/}$mice had a significantly lower total histological score at 8 days postoperatively compared with WT mice. (c,d) Cell density in the healing region was significantly lower in $\mathrm{Tnmd}^{-1-}$ versus WT mice. DAPI images were analyzed by computerized image analysis with ImageJ. (e) Ectopic endochondral ossification was not revealed by safranin $\mathrm{O}$ staining in the tendons of either genotype at day 8 . (f-h) In HE-stained sections increased areas of adipocyte accumulation and numbers of large blood vessels were detected in the scar region of Tnmd $^{-/}$tendons compared with WT mice. (i) Visualization of adipocytes and blood vessels in Tnmo ${ }^{-1}$ and WT Achilles tendon scars via immunofluorescence staining for perilipin and collagen IV. (j,k) The perilipin-positive areas and number of collagen IV-labeled blood vessels were significantly higher by 8 days after surgery in Tnmd $^{--}$versus WT mice. (I-n) qRT-PCR revealed upregulated mRNA levels of Ppary and Lpl, but no changes in Fabp4 expression in Tnmd ${ }^{/-}$versus WT tendons. For quantification in (b, d, g, h, j and $\mathbf{k}$ ), statistical significance was calculated using two-tailed non-parametric Mann-Whitney test, $n=8$ (8 animals per group; each animal represented by 3 tissue sections). For qRT-PCR in (I, $\mathbf{m}$ and $\mathbf{n}$ ), statistical significance between 2 groups was determined by unpaired Student's $t$-test (two-tailed) for 5 independent experiments. ${ }^{\star} P<0.05$; ${ }^{* \star} P<0.001$, compared with WT. S, scar; T, tendon; yellow arrows, blood vessels; black arrows, adipocytes. Scale bars: $200 \mu \mathrm{m}$

(Figures 1a and b). Quantitatively, total cell density was significantly lower in the $\mathrm{Tnmd}^{-1}$ mice at 8 postoperative days (Figures 1c and d). Ectopic ossification after tenotomy of rodent Achilles tendon at late stages of the tendon healing process has been reported in previous studies. ${ }^{18-20} \mathrm{How}-$ ever, ectopic endochondral ossification was not detected in the scar tissues in either of the genotypes following safranin $\mathrm{O}$ staining at 8 days post-injury (Figure 1e). In contrast, the mean area of adipocyte accumulation, the number of blood vessels observed in HE staining analyses (Figures $1 \mathrm{f}-\mathrm{h}$ ) and validated by immunofluorescence staining and quantification for perilipin- (Figures 1i and j) and collagen IV-positive areas (Figures $1 \mathrm{i}$ and $\mathrm{k}$ ), were significantly increased in the scar sites of $\mathrm{Tnmd}^{-1-}$ mice compared with WT controls. We also found increased mRNA levels of the adipogenic marker genes, peroxisome proliferator-activated receptor gamma (Ppary) and lipoprotein lipase $(L p l)$ in the tendons of $\mathrm{Tnmd}^{-1}$ mice through quantitative reverse transcriptase PCR (qRT-PCR) (Figures $1 \mathrm{l}$ and $\mathrm{m}$ ). Expression of fatty acid-binding protein 4 (Fabp4), another adipogenic marker, was unaffected (Figure 1n). The above data revealed for the first time that the absence of Tnmd leads to an inferior morphological outcome and lower cellular density, whilst it activates adipocyte accumulation and adipose-related gene expression as well as vessel numbers in the early repair region of injured tendons.

$\mathrm{Tnmd}^{-1-}$ tendons demonstrate reduced cell proliferation and CD146-positive cell numbers, downregulated levels of certain tendon-related genes, whilst increasing cell apoptosis and occurrence of p53-expressing cells. To test whether the reduction in cell numbers was due to a decreased proliferation or increased apoptosis, we carried out proliferative and apoptotic assays by bromodeoxyuridine (BrdU) and terminal deoxynucleotidyl transferase-mediated dUTP-biotin nick end labeling (TUNEL) stainings. Furthermore, immunofluorescence staining was also performed for p53, which regulates the apoptosis in oxidative stressexposed tenocytes, ${ }^{21}$ and has been previously shown by us to be elevated in the tendons of uninjured $\mathrm{Tnmd}^{-1-}$ mice. ${ }^{11}$ BrdU analysis confirmed a lower number of proliferating cells at the scar site of injured Achilles tendons in $\mathrm{Tnmd}^{-1-}$ than WT mice (Figures $2 a$ and b). Furthermore, TUNEL assays and immunofluorescence staining for p53 showed that Tnmd $^{-1}$ scars had an increased number of apoptotic cells (Figures 2c-f). In order to track activated local stem/ progenitor cells at the scar site, we performed immunofluorescence analysis for CD146, which labels MSCs as well as the TSPCs. ${ }^{22-24}$ The number of CD146-positive cells was significantly lower in $\mathrm{Tnmd}^{-1}$ compared with WT mice eight days after injury (Figures $2 \mathrm{~g}$ and $\mathrm{h}$ ). Following this, we analyzed how the absence of Tnmd affects the expression levels of tendon-associated gene markers using qRT-PCR of $\mathrm{Tnmd}^{-/}$and WT tendon-derived mRNA. We observed significantly lower mRNA levels for early growth response protein 1 and 2 (Egr1, Egr2), collagens I, III and V (Col1a1, Col3a1, Col5a1), tenascin C (Tnc), thrombospondin 2 (Thbs2), alpha smooth muscle actin (Acta2) and transforming growth factor beta 1 (Tgfb1) in $\mathrm{Tnmd}^{-/}$samples (Figure 2i). On the contrary, the relative expression levels of mohawk $(M k x)$, scleraxis $(S c x)$, cartilage oligomeric protein (Comp) and lubricin (Prg4) displayed a dramatic increase, without affecting those of sine oculis homeobox homolog 1 (Six1), collagens VI and XII (Col6a1, Col12a1) and thrombospondin 4 (Thbs4) (Figure 2i). In sum, we concluded that the loss of Tnmd causes simultaneously reduced numbers of BrdU- and CD146-expressing cells, but an increased incidence of TUNEL- and p53-positive cells in the tendon scar tissue and dysregulated expression of key tendon-related transcription factors and ECM genes, which in turn can lead to altered tendon tissue composition during repair.

$\mathrm{Tnmd}^{-/}$scar tissues are characterized by erroneous ECM deposition and abnormal macrophage profile. The ECM of tendon tissue is composed primarily of collagen I, as well as collagen III, elastin and various proteoglycans and mucopolysaccharides. ${ }^{3}$ Anomalies in the ECM composition of the scar tissue after tendon injury may contribute to a poor and delayed healing process resulting in compromised tissue quality. $^{20}$ Prompted by this observation and the gene expression changes in $\mathrm{Tnmd}^{-1}$ tendons, we carried out an ECM phenotyping of the scar tissues of both genotypes. First, we performed immunofluorescence staining with an anti-Cterminal Tnmd antibody visualizing Tnmd secretion in the ECM of WT mice Achilles tendon, but not in $\mathrm{Tnmd}^{-/}$mice (Figures $3 a$ and b). Surprisingly, three ECM proteins, namely biglycan, Comp and fibronectin, were more expressed in Tnmo $^{-1}$ tendon healing sites than WT mice (Figures 3c-h). The increased protein deposition of Comp in $\mathrm{Tnmd}^{-1}$ samples was consistent with the qRT-PCR data showing higher Comp mRNA levels in this group (Figure 2i). However, collagens I and III, decorin, elastin, fibromodulin and lumican were not significantly affected (Supplementary Figure 1a). Nonetheless, picrosirious red-stained tendon sections analyzed by polarized light microscopy exhibited ECM containing thicker collagen fibers in the scar areas and tendon ends of 


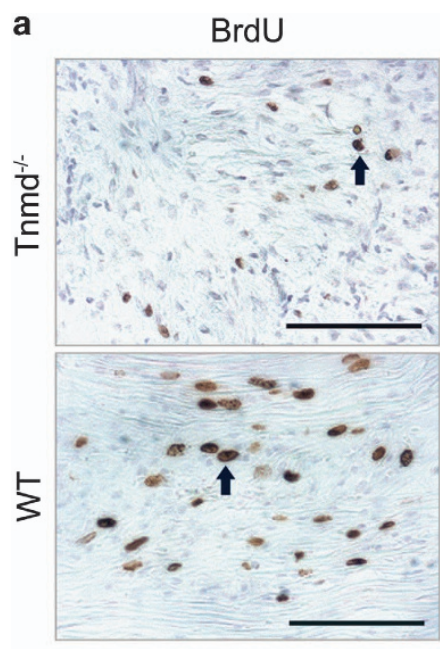

c TUNEL/DAPI
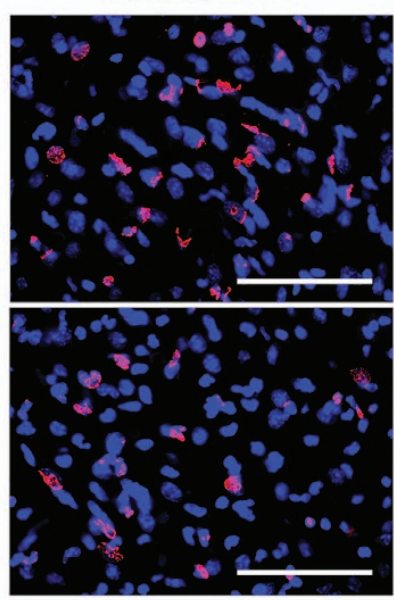

d

b

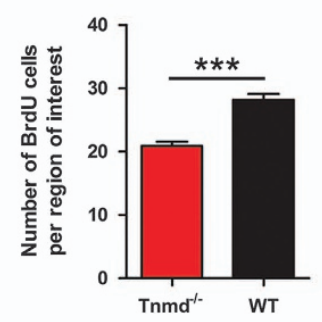

e
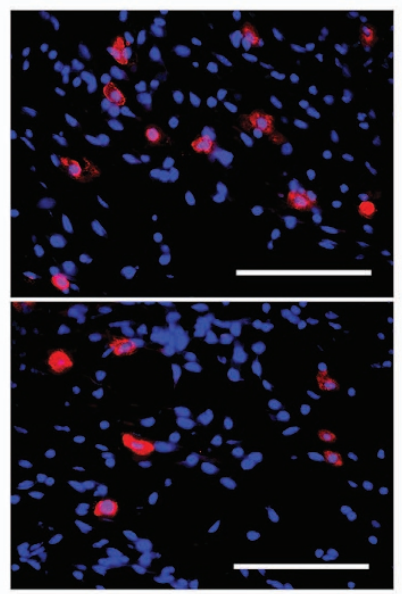

f

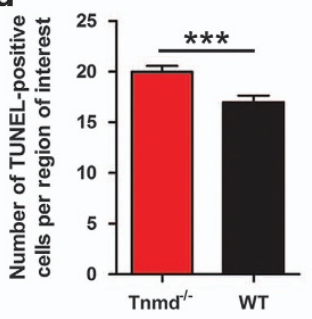

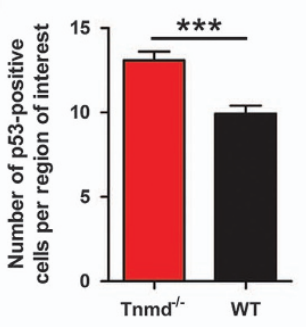

g CD146/DAPI
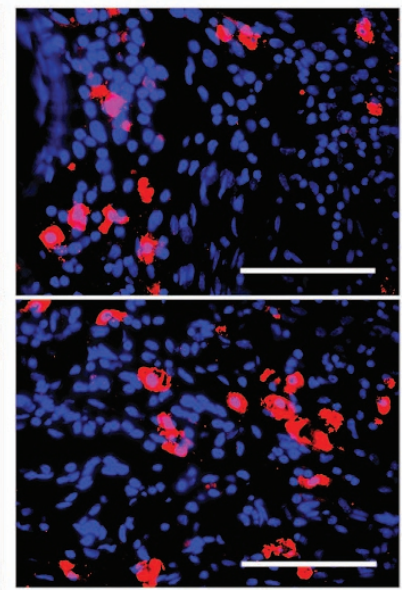

h

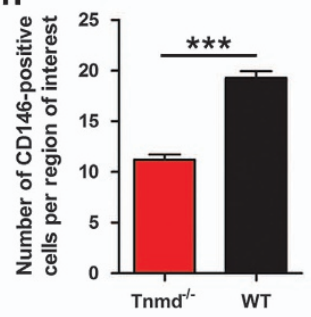

i

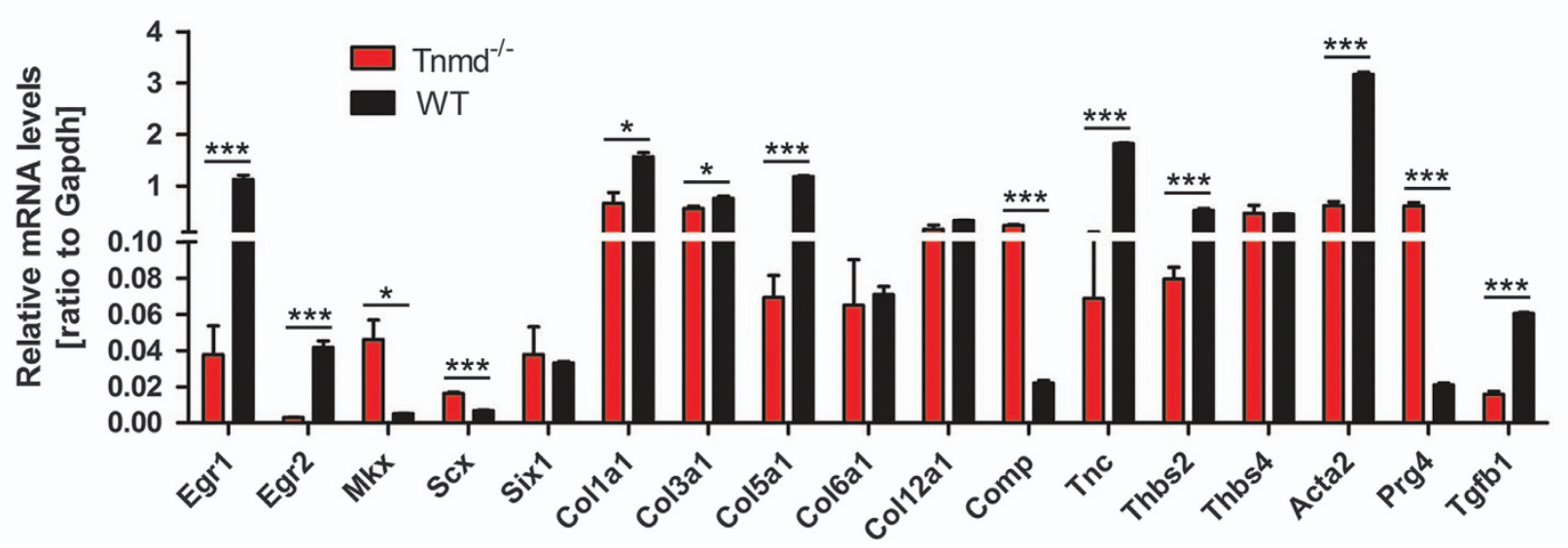

Figure 2 Tnmd deficiency results in reduced cell proliferation, CD146-positive cells, increased cell apoptosis and p53-expressing cells and an altered expression of certain tendon-related genes. $(\mathbf{a}, \mathbf{b})$ BrdU staining and quantification in the tendon scars revealed decreased proliferative cell numbers in Tnmo ${ }^{-1}$ versus WT mice at 8 days postoperatively. (c,d) TUNEL-based analyses detecting apoptotic cells showed increased cell apoptosis in Tnmo ${ }^{-1}$ mice compared with WT mice. (e,f) Increased number of p53positive cells was found at day 8 in the tendons scar tissues of $\mathrm{Tnmd}^{-1}$ tendons compared with WT mice. (g,h) Immunofluorescence staining for CD146 showed that the number of CD146-expressing cells, corresponding to local MSCs and/or TSPCs was lower in Tnmd ${ }^{-1}$ mice than WT mice. (i) $\mathrm{Tnmd}^{-1-}$ tendons displayed significantly lower expression levels for Egr1, Egr2, Col1a1, Col3a1, Col5a1, Tnc, Thbs2, Acta2 and Tgfb1 compared with WT mice. In contrast, the relative gene expression of Mkx, Scx, Comp and Prg4 displayed a dramatic increase. No effect was found for Six1, Col6a1, Col12a1 and Thbs4. For quantification in (b, d, $\mathbf{f}$ and $\mathbf{h})$, statistical significance was calculated using twotailed non-parametric Mann-Whitney test, $n=8$ ( 8 animals per group; each animal represented by 3 tissue sections). For qRT-PCR in $\mathbf{i}$, statistical significance between 2 groups was determined by unpaired Student's $t$-test (two-tailed) for 5 independent experiments. ${ }^{*} P<0.05 ;{ }^{* *} P<0.001$, compared with WT. Black arrows, BrdU-positive cells. Scale bars: $100 \mu \mathrm{m}$

the $\mathrm{Tnmd}^{-/}$than that of WT mice (Supplementary Figure 1b). Our observation of the increased erroneous ECM deposition in the repair sites of $\mathrm{Tnmd}^{-1}$ tendons motivated us to investigate by using target-specific ELISA whether $\mathrm{Tnmd}^{-/}$TSPCs secrete higher amounts of biglycan and fibronectin proteins. The obtained quantitative data further confirmed our in vivo results by showing that the levels of both proteins were significantly increased in the supernatant of $\mathrm{Tnmd}^{-/}$compared with WT TSPCs (Figures 3i-l).

The repair of injured tendons begins with an early inflammatory response that is associated with infiltration of pro-inflammatory, classically activated (M1) macrophages, whereas the secondary inflammatory response involves 
a
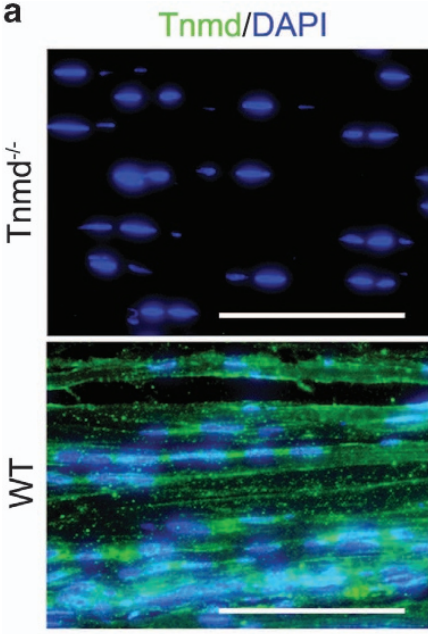

b

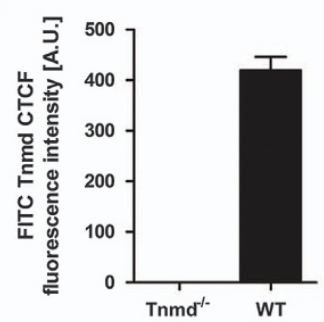

i

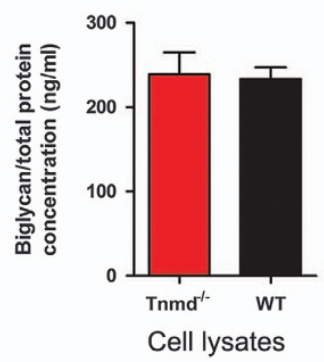

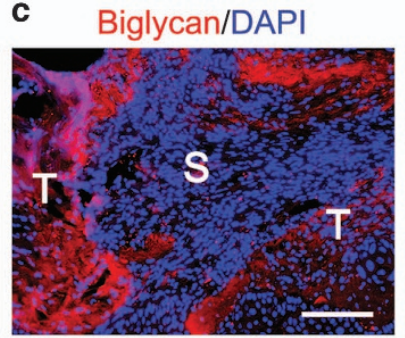

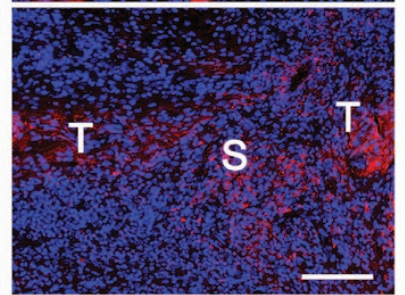

d
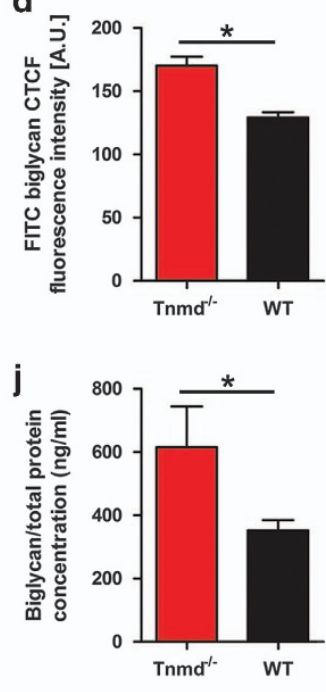

Supernatants

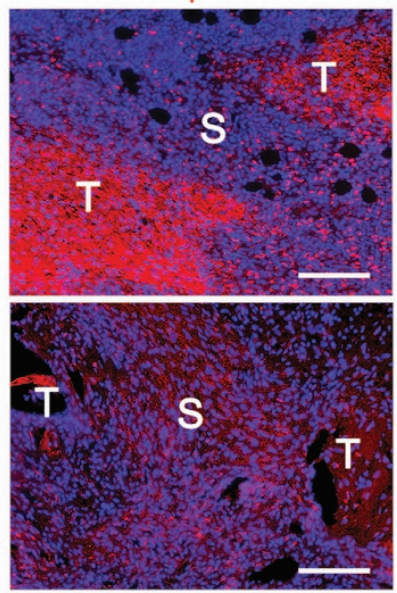

f

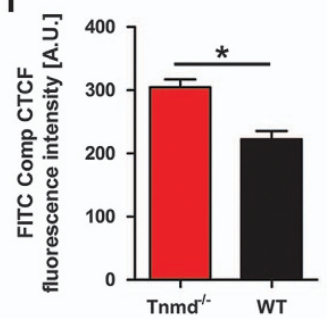

k

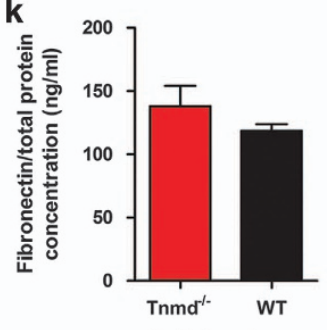

Cell lysates g Fibronectin/DAPI
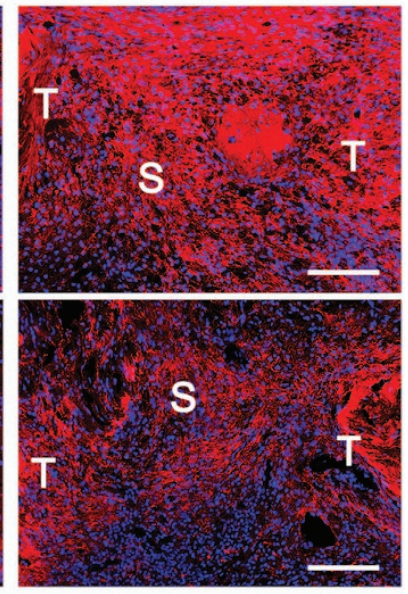

h
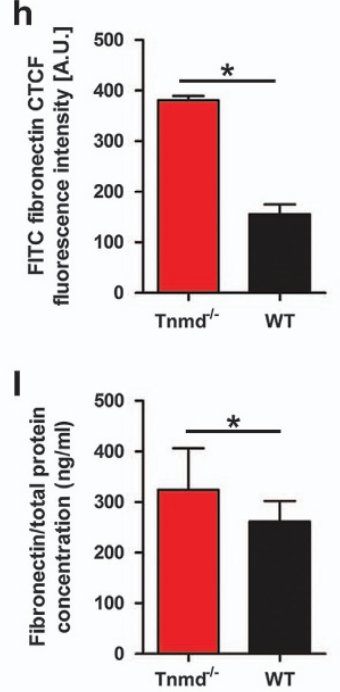

Supernatants

Figure 3 The absence of Tnmd increases erroneous ECM deposition. (a,b) Immunofluorescence staining with anti-Tnmd C-terminal antibody showed Tnmd secretion in the ECM of WTAchilles tendon, but not in $\mathrm{Tnmd}^{-/}$. (c-h) Biglycan, Comp and fibronectin protein deposition in the tendon scar, analyzed by fluorescent digital signal quantification, was clearly augmented in $\mathrm{Tnmd}^{-1}$ when compared with WT mice at 8 days postoperatively. (i-l) Biglycan and fibronectin protein levels from cell lysates and supernatant were

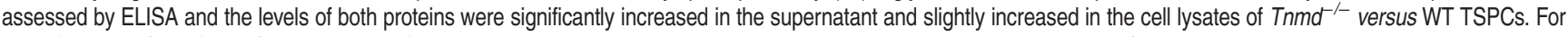
quantification in ( $\mathbf{b}, \mathbf{d}, \mathbf{f}$ and $\mathbf{h})$, statistical significance was calculated using two-tailed non-parametric Mann-Whitney test, $n=3$ ( 3 animals per group; each animal represented by 3 tissue sections). For ELISA in (i, j, $\mathbf{k}$ and I), statistical significance between 2 groups was determined by unpaired Student's t-test (two-tailed) for two independent experiments with two donors/genotypes. ${ }^{*} P<0.05$ compared with WT. S, scar; T, tendon ends. Scale bars: $100 \mu \mathrm{m}$

anti-inflammatory, alternatively activated (M2) macrophages. ${ }^{25}$ Recent evidence suggested that proper modulation of inflammation in the early stages of tendon repair may lead to improved healing. ${ }^{26}$ Interestingly, in our model, the numbers of cells positive for CD68, (a prominent surface marker for M1 and tissue-resident macrophages), and CD80, (a M1 macrophage surface marker), were significantly increased (Figures $4 a-d)$, whilst the number of cells expressing CD163, (a M2 macrophage surface marker), was significantly reduced in $\mathrm{Tnmd}^{-1-}$ mice compared with WT mice eight days after injury (Figures $4 \mathrm{e}$ and f). These results were further substantiated by immunofluorescence staining of $F 4 / 80$, a monoclonal antibody directed specifically against mouse macrophages, demonstrating a significant increase of labeled cells in the scar sites of $\mathrm{Tnmd}^{-1}$ mice (Figures $4 \mathrm{~g}$ and $\mathrm{h}$ ). Collectively, this set of data shows that Tnmd deficiency leads to erroneous ECM deposition in vivo and in vitro and leads to an abnormal macrophage profile with pre-dominating M1 macrophages in the repair site at day 8 of tendon healing.

Tnmd $^{-1}$ TSPCs show significantly lesser migratory and proliferative capacities but have accelerated adipogenic differentiation rate and significantly upregulated Ppar and $L p l$ mRNA expression. Figures $1 \mathrm{c}$ and $\mathrm{d}$ and Figures $2 \mathrm{a}, \mathrm{b}, \mathrm{g}$ and $\mathrm{h}$ demonstrated that loss of Tnmd is associated with significantly lower cell density and numbers of BrdU- and CD146-positive cells in the scar tissues at day 8 . These imply that Tnmd may regulate the migration and 
a
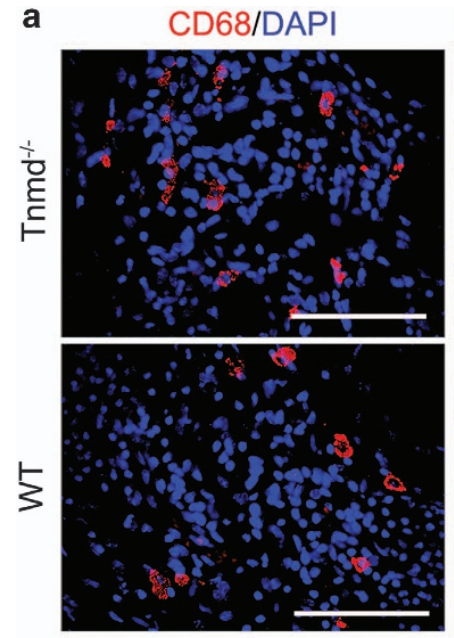

b

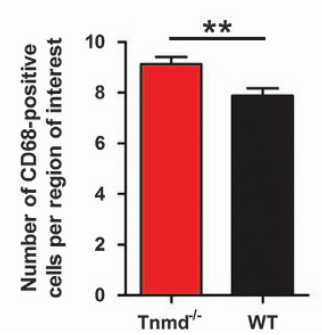

c CD80/DAPI
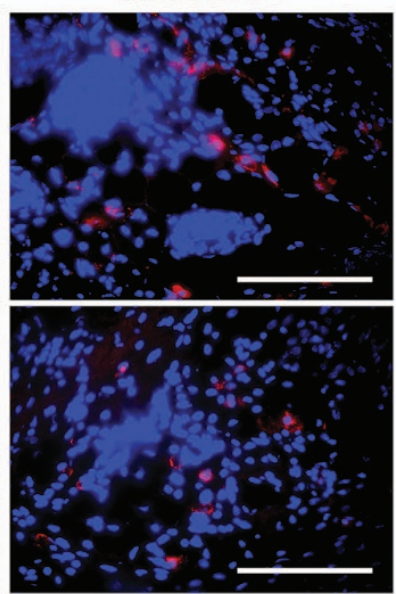

d

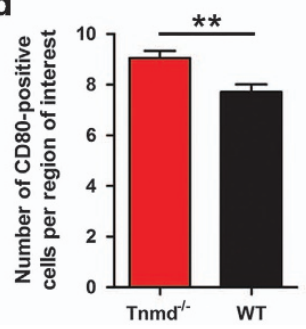

e
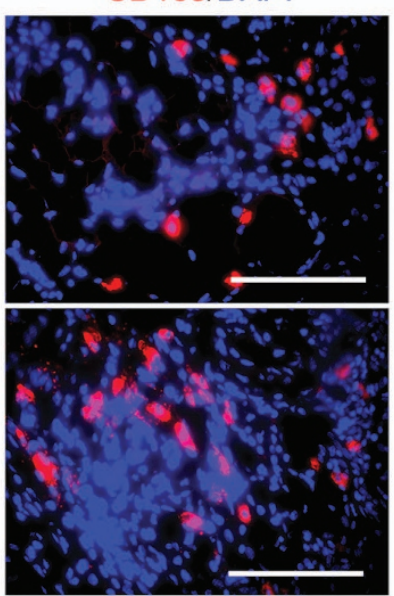

f

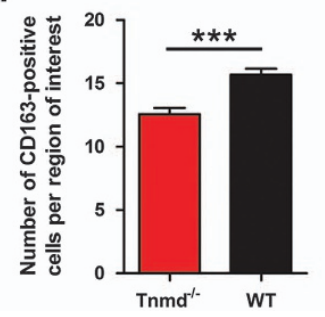

g F4/80/DAPI
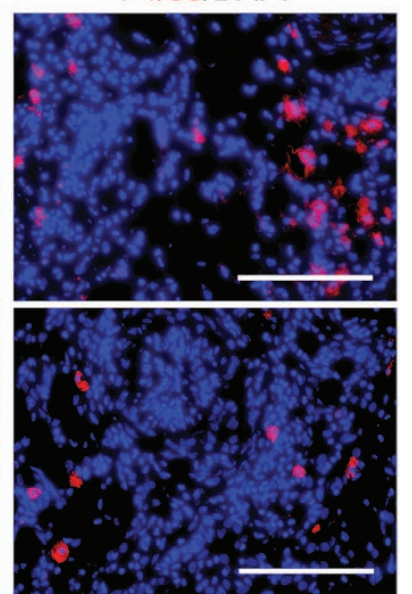

h

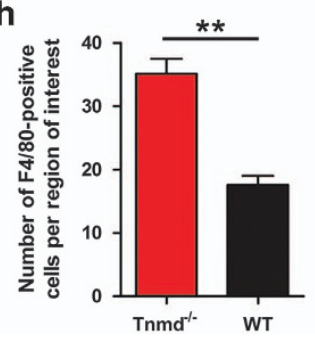

Figure 4 The lack of Tnmd alters the macrophage profile during early tendon healing. (a-h) The numbers of CD68-, CD80- and F4/80-positive cells were significantly increased, whereas the number of CD163-positive cells was significantly reduced in the $\mathrm{Tnm}^{-/}$tendon scar tissues compared with WT mice. For quantification in (b, d, $\mathbf{f}$ and $\mathbf{h}$ ), statistical significance was calculated using two-tailed non-parametric Mann-Whitney test, $n=8$ ( 8 animals per group; each animal represented by 3 tissue sections). ${ }^{\star \star} P<0.01$; ${ }^{* * *} P<0.001$, compared with WT. Scale bars: $100 \mu \mathrm{m}$

self-renewal capacities of TSPCs. Therefore, we carried out scratch assays mimicking wound closure in vitro. To estimate the effect of ECM proteins and because of the increased fibronectin deposition in $\mathrm{Tnmd}^{-/}$tendons, our scratch assays were performed on collagen I- and fibronectincoated dishes. Quantification of scratch closure rate after $24 \mathrm{~h}$, showed that the migration speed of $\mathrm{Tnmd}^{-1}$ TSPCs was significantly lower compared with WT TSPCs (Figures $5 a-d)$. This finding was further solidified by random migration analysis after $48 \mathrm{~h}$, in which forward migration index (FMI) of multiple single cells migrating on either of the ECM proteins was calculated (Figures $5 e$ and f). Quantification of velocity, accumulated and Euclidean distance, also clearly indicated a significant reduction of $\mathrm{Tnmd}^{-1}$ TSPCs motility compared with WT (Figures $5 \mathrm{~g}-\mathrm{l})$. Furthermore, during 12 days of culture, DNA-based CyQUANT assays at various time points showed that $\mathrm{Tnmd}^{-1}$ TSPCs proliferated significantly slower than WT TSPCs (Figure $5 \mathrm{~m}$ ), confirming and expanding our earlier report that Tnmd is a positive regulator of TSPC self-renewal. ${ }^{13}$

The observed adipocyte accumulation during early tendon healing prompted us to test whether the loss of Tnmd can accelerate TSPCs differentiation into adipocytes. Previously, we have observed an increased tendency of in vitro adipogenesis of $\mathrm{Tnmd}^{-1-}$ TSPCs. ${ }^{13}$ Here we again subjected TSPCs to adipocyte differentiation and examined the outcome in-depth. $\mathrm{Tnmd}^{-1-}$ TSPCs grown in adipogenic medium had significantly more BODIPY 493/503 staining of neutral lipid droplets, indicating a higher adipogenic propensity than WT TSPCs after 7, 14 and 21 days, respectively (Figures $6 a$ and b). Additional analysis, with the AdipoRed reagent revealed similar results (Figure 6c). Consistent with our in vivo results, semi-quantitative RT-PCR and densitometric PCR band evaluation showed that the expression levels of Ppary and $L p l$, but not Fabp4, were significantly increased in $\mathrm{Tnmd}^{-/}$ TSPCs compared with those of WT following 21 days of adipogenic stimulation (Figures $6 \mathrm{~d}-\mathrm{f}$ ). We conclude that the lack of Tnmd in TSPCs negatively alters their migratory and proliferative capacities, whilst accelerating their commitment towards adipocytes and the expression of critical adipose regulatory genes such as Ppary and $L p l$.

\section{Discussion}

Effective strategies to speed up the healing process of tendon injuries are still not developed because the understanding of tendon biology lags far behind that of the other components of the musculoskeletal system, and the molecular mechanisms controlling the migration, proliferation and fate of TSPCs during tendon repair are not well understood. ${ }^{1,9}$ Therefore, it is still very challenging to identify molecular targets that can be used to develop medicinal boosters for complete and timely repair of injured tendons or ligaments. Tnmd is a useful phenotypic marker of mature tenocytes and ligamentocytes that has been shown to have intriguing and diverse roles in developing tendons and those challenged by physical 


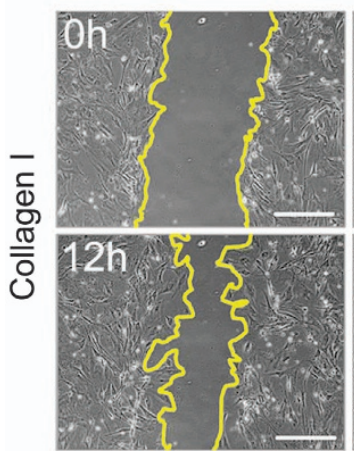

b

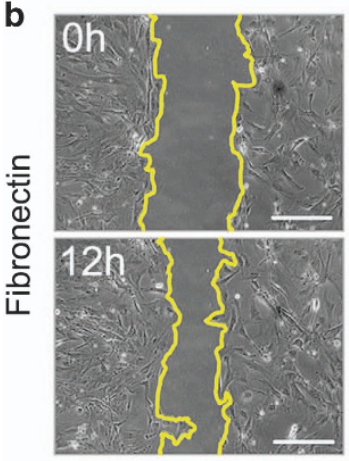

WT
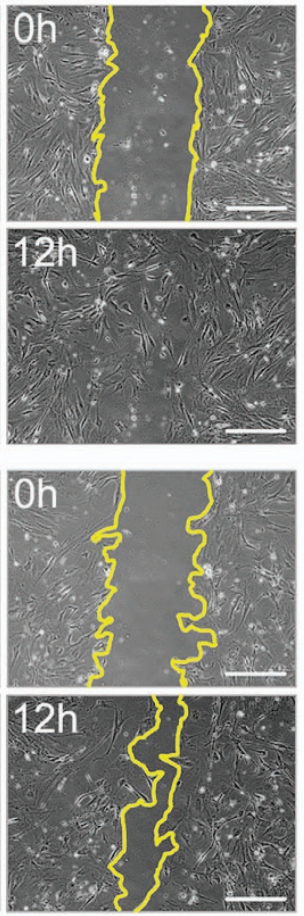

e

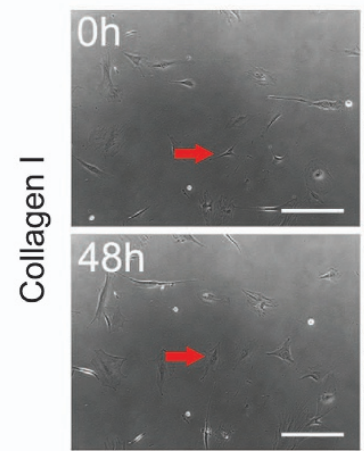

f

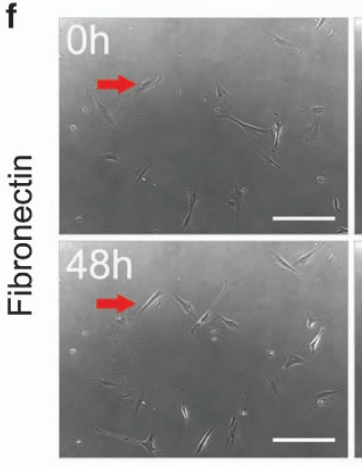

WT
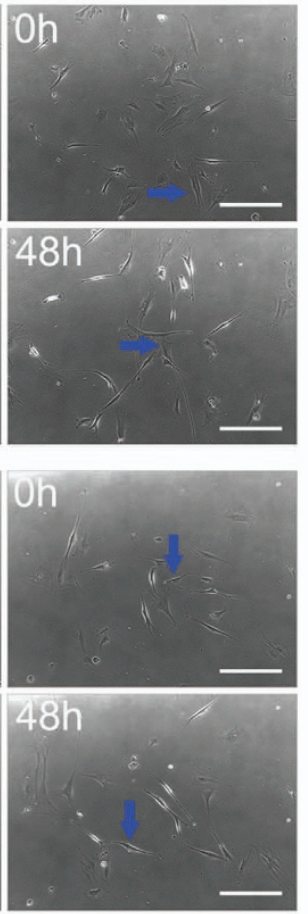

FMI
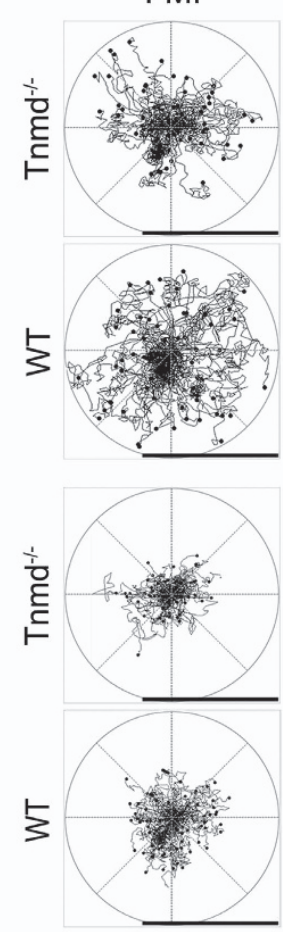

C

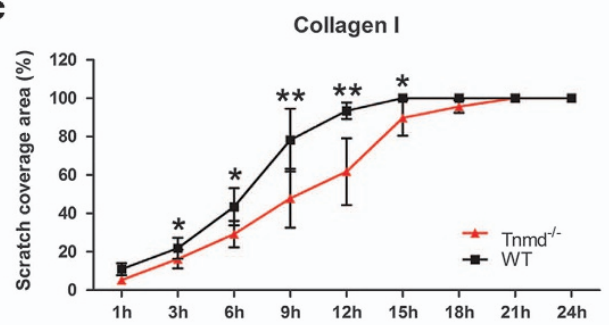

d

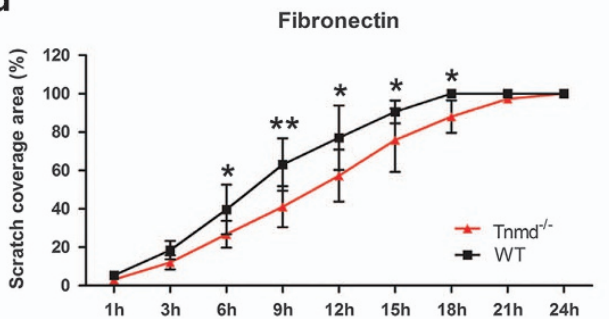

g

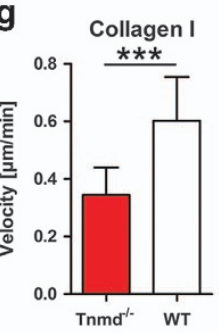

k

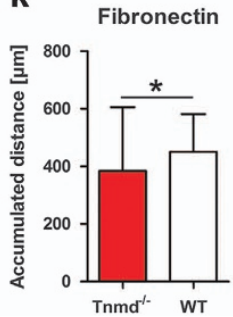

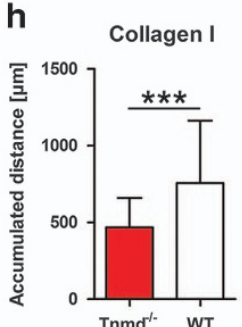

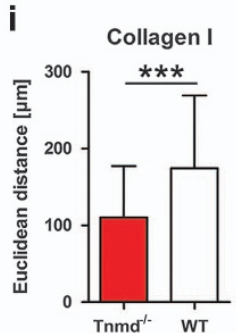

j
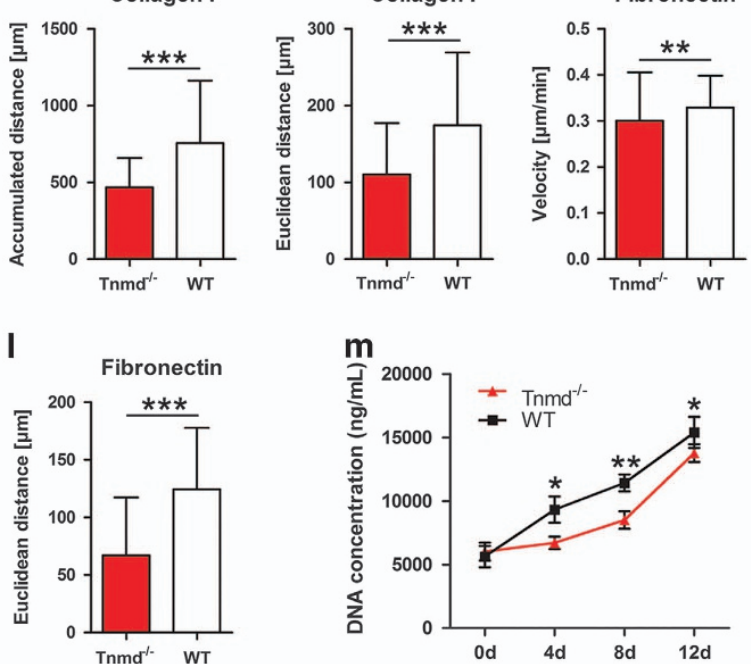

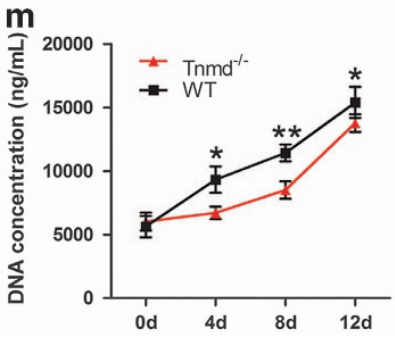

Figure 5 The absence of Tnmd in TSPCs leads to significantly reduced cell migration and proliferation. (a-d) In vitro wound healing assays on collagen I and fibronectin showed that $\mathrm{Tnmd}^{-1-}$ TSPC scratch closure was significantly slower compared with WT TSPCs. The borders of the scratches are outlined with yellow lines. (e,f) Forward migration index (FMI) plots showed that $\mathrm{Tnmd}^{-/}$TSPCs were indeed less migratory than WT TSPCs. Upper arrows on each type of matrix show the start point while lower arrows the end point of example migratory cells. ( $\mathbf{g}-\mathbf{I})$ Quantification of velocity, accumulated and Euclidean distances further validated Tnmd $^{-1}$ migratory deficiency. ( $\mathbf{m}$ ) During

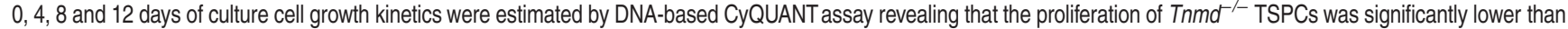
that of WT TSPCs. For quantification in (c, $\mathbf{d}$ and $\mathbf{m}), n=4$ independent experiments per group. For quantifications in random migration, $n=3$ independent experiments per group (total of 70-80 tracks per genotype). Statistical significance was calculated using two-tailed non-parametric Mann-Whitney test. ${ }^{*} P<0.05 ;{ }^{* *} P<0.01 ;{ }^{* * *} P<0.001$, compared with WT. Blue arrows, WT TSPCs; d, day; h, hour; Red arrows, Tnmd ${ }^{-1}$ TSPCs; Scale bars: $200 \mu \mathrm{m}$

exercise..$^{9-12,27}$ Herein, we further explored the potential roles of Tnmd in the tendon healing process by subjecting $\mathrm{Tnmd}^{-/}$ mice to full thickness Achilles tendon injury and carrying out indepth characterization of the scar tissues at day 8 as well as investigating certain TSPCs functions in vitro. The novel results of this study demonstrate for the first time that the absence of Tnmd causes inferior tendon repair process, as shown by adipocyte accumulation and fibrovascular scar formation during early tendon healing.

Efficient molecular modulation of tendon healing should accelerate cell proliferation and inhibit apoptosis, or at least not augment the number of apoptotic events. ${ }^{28}$ We have 
a
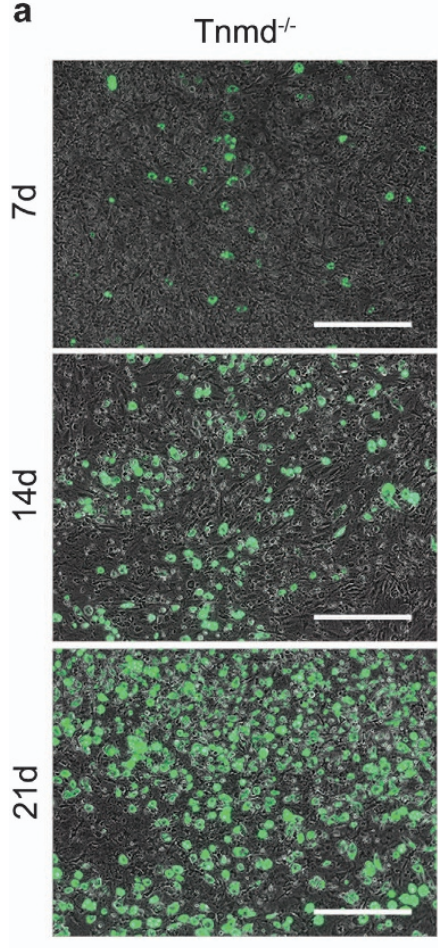

d

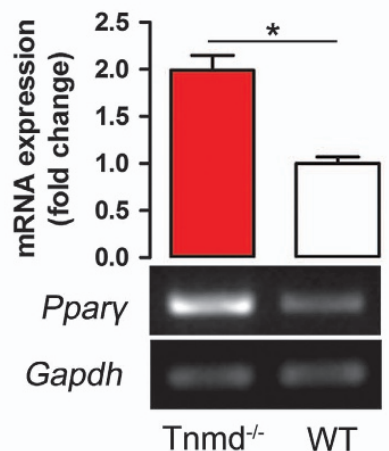

WT
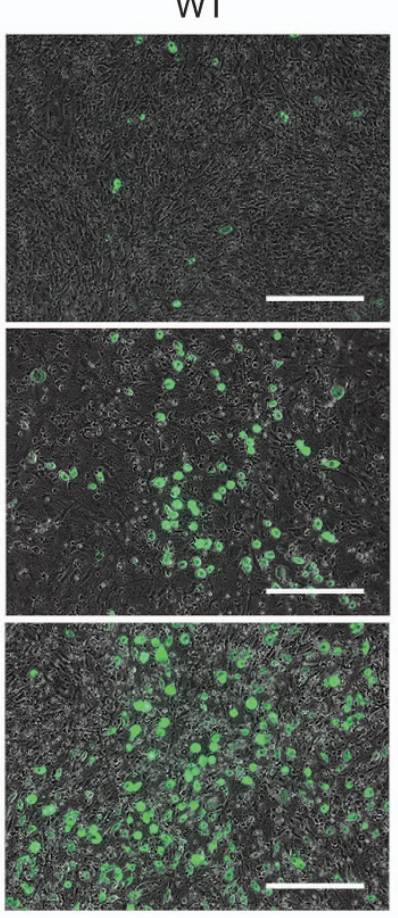

b
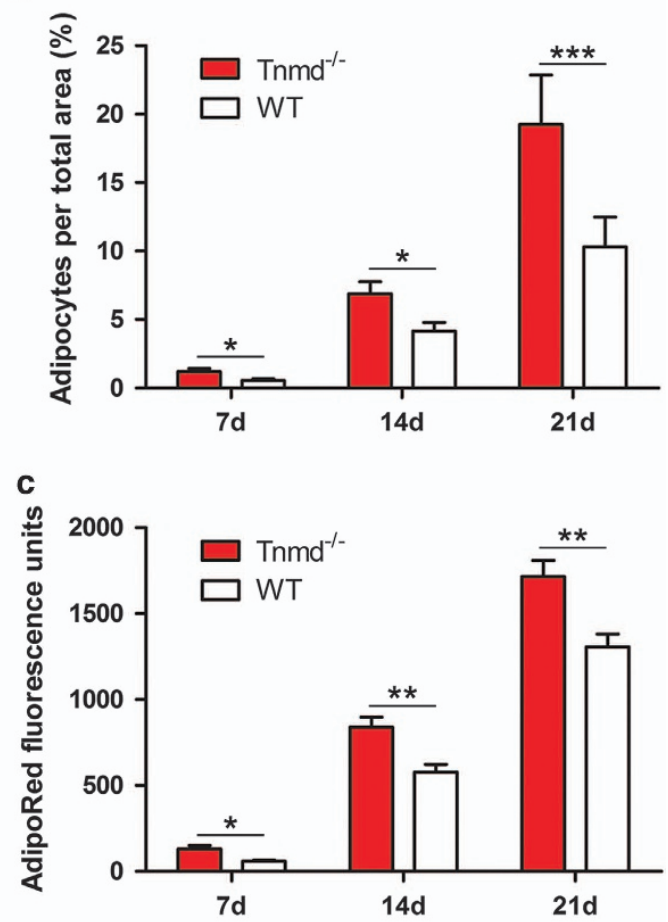

f

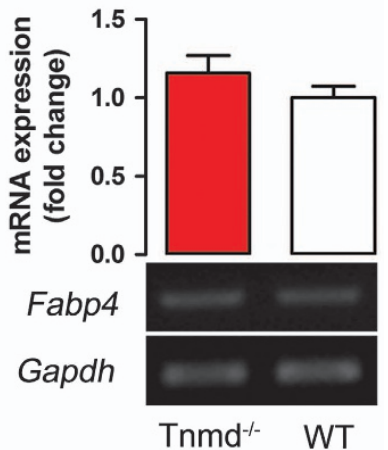

Figure 6 The absence of Tnmd in TSPCs leads to significantly accelerated adipogenic differentiation and upregulated Ppary and $\mathrm{Lpl}^{\text {expression levels. (a,b) Tnmd }}{ }^{-1}$ TSPCs grown in adipogenic medium had significantly more BODIPY 493/503 staining of neutral lipid droplets, indicating a higher propensity of these cells to form adipocytes than WT TSPCs after 7, 14 and 21 days, respectively. (c) AdipoRed fluorescent quantitative assay confirmed these results. (d-f) Semi-quantitative RT-PCR and densitometric band analysis revealed that the expression of the two adipogenic marker genes Ppary and Lp/ was increased, while Fabp4 levels were not significant changed in Tnmo ${ }^{-1}$ TSPCs after $^{-}$ 21 days of adipogenic differentiation versus WT TSPCs. For quantification in $\mathbf{b}$ and $\mathbf{c} n=4$ independent experiments. Statistical significance was calculated using two-tailed nonparametric Mann-Whitney test. For semi-quantitative RT-PCR, $n=5$ independent experiments per group. Statistical significance between 2 groups was determined by unpaired Student's $t$-test (2-tailed). ${ }^{*} P<0.05 ;{ }^{* *} P<0.01 ;{ }^{* \star *} P<0.001$, compared with WT. Scale bars: $200 \mu \mathrm{m}$

already reported that the tendons of Tnmd knockout mice exhibit reduced cell density and proliferation ${ }^{11}$ concomitant with apparent an in vitro phenotype of $\mathrm{Tnmd}^{-1}$ TSPCs, which were significantly less self-renewing, and more senescent. ${ }^{13}$ Our current study provides further evidence that the loss of Tnmd expression in the healing tendon results in reduced cell density and proliferation and lower numbers of CD146expressing cells, as well as augmented cell apoptosis and higher numbers of p53-positive cells. Furthermore, we show for the first time that Tnmd $^{-1-}$ TSPCs also have significant migratory deficits in two different experimental set-ups. Hence, we suggest that Tnmd has anti-apoptotic and anti-senescence roles and has important regulatory roles in cell migration and proliferation during the early stage of tendon repair. The positive association of Tnmd expression with advancement of tendon healing was previously suggested by Tokunaga et al. ${ }^{29}$ in a growth factor-dependent model of rotator cuff healing.

Adipocyte accumulation and fibrovascular scar are common pathological changes that occur in ruptured tendons and ligaments. ${ }^{30-34}$ They often do not properly remodel and in some cases continue to worsen even after surgical repair and physiotherapy. However, little is known of the pathophysiological pathways behind these phenomena. ${ }^{30-34}$ Persistent or unresolved inflammation is considered a major trigger in many fibrotic diseases and in tendon healing has been associated with abnormal fibrogenesis. ${ }^{35-37}$ Recent in vivo large animal 
studies showed that inflammatory factors are dramatically upregulated within the first week after tendon injury, which in turn stimulate the production of proteases, cause apoptosis of tendon cells, impede the intrinsic repair process, and promote adhesion formation. ${ }^{38-42}$ During inflammation, macrophages have essential roles in both promoting and resolving inflammation and in both facilitating and modulating tissue repair. In an injury setting, M1 cells predominate early, whereas M2 cells accumulate later. Hence, in tendon injury, it could be postulated that M1 macrophages promote repair by stimulating ECM production and that M2 macrophages enter the process to repress inflammation and clear excess ECM, a concept that is consistent with experimental evidence. ${ }^{26}$ Disturbing the balance between these macrophage subtypes may result in abnormal scar formation, a defective repair process and impaired tissue function. ${ }^{26}$ In our model, Tnmd $^{-1}$ scars at day 8 exhibited higher numbers of vessels than WT scars, and displayed a macrophage profile of predominantly M1 cells with lower M2 numbers. This finding may help to explain the excess ECM protein deposition of biglycan, Comp and fibronectin, seen in $\mathrm{Tnmd}^{-/}$tendons. Similarly, some recent reports dealing with tendon healing demonstrated higher expression of biglycan and Comp in the ectopic chondro-ossification sites in injured tendon tissues. ${ }^{20,43}$ In addition, Spiegelman et al. ${ }^{44}$ found that fibronectin protein can regulate adipogenic gene expression. Furthermore, a study focusing on stem cell-based therapy of tendon injuries suggested that a lower M2 macrophage number leads to less accumulation of CD146-positive cells and more erroneous matrix deposition at the repair site. ${ }^{45}$ Thus, we suggest that the absence of Tnmd leads to enhanced vascular invasion, a prolonged inflammatory response, aggravated deposition or delayed clearing of excessive erroneous ECM. A deficiency of our study is that we do not describe the precise molecular mechanism by which Tnmd regulates the above processes. At present, we are unable to elucidate the exact Tnmd mode of action because we do not yet know the binding partners of this protein. Future studies are needed if we are to decipher the Tnmd signaling pathways. It will also be important to compare our small animal model with clinical tendon pathologies, for example by investigating Tnmd expression levels in different tendinopathy forms.

Interestingly, many recent studies have focused on understanding Tnmd involvement in obesity and diabetes. ${ }^{46-52}$ Of interest, Senol-Cosar et al. ${ }^{46}$ suggested that Tnmd facilitates pre-adipocyte terminal differentiation while Jiang et al. ${ }^{14}$ showed that overexpression of Tnmd actually inhibits adipogenesis of murine MSCs. These data suggest that Tnmd might have cell type-specific modes of action; a suggestion reinforced by the contrasting observations that Tnmd causes proliferation of tendon-derived cells but inhibits proliferation of vascular-derived cells. ${ }^{9}$ Our results are in line with the study of Jiang et al. ${ }^{14}$ as we showed $\mathrm{Tnmd}^{-/}$scars had significantly higher adipocyte accumulation and also that $\mathrm{Tnmd}^{-/}$TSPCs had a higher rate of differentiation into adipocytes. We propose that the different regulatory mechanisms of the Tnmd signaling pathway are involved in different cell types, which will be revealed when the Tnmd molecular network is finally mapped.

Ppary is a transcriptional master regulator of adipogenic differentiation and stimulates adipogenesis. ${ }^{53-56}$ Here, we showed that concomitantly with the higher adipocyte numbers, the lack of Tnmd significantly enhanced the expression levels of Ppary and $L p l$ in vivo and in vitro. Furthermore, we observed that the absence of Tnmd results in dysregulated expression of key tendon transcription factors and ECM genes and proteins, which in turn may lead to altered scar composition and thereby increased lipid accumulation. Gehwolf et al. ${ }^{57}$ revealed that loss of the expression of the ECM protein Sparc drives adipocyte differentiation in tendons. Our study does not differentiate whether the pathways underlying the induction of adipogenesis either by Ppary upregulation or by changes in ECM properties operate independently of each other or in an interdependent manner. ${ }^{57,58}$ We can not provide a conclusive answer to this question, but we propose that Tnmd may strongly influence adipogenesis during tendon healing through the regulation of Ppary expression, ECM composition and/or by preventing TSPC adipocyte commitment.

In summary, we created an Achilles tendon injury model in $\mathrm{Tnmd}^{-1}$ mice, that showed that loss of Tnmd results in inferior tendon repair characterized by increased adipocyte accumulation, reduced cell density, proliferation and CD146-positive cells, increased apoptotic and p53-expressing cells, M1:M2 macrophage ratio changes, abnormal expression of tendonrelated genes, and augmented fibrovascular scar composition. Concomitant in vitro analysis of $\mathrm{Tnmd}^{-1-} \mathrm{TSPC}^{-}$revealed significantly reduced migratory and proliferative capacities, but upregulated adipogenic gene marker levels and accelerated differentiation down this lineage. Thus, our results suggest that Tnmd is required for prevention of adipocyte accumulation and fibrovascular scar formation during the early phase of tendon healing.

\section{Materials and Methods}

Animal model and surgical procedure. $\mathrm{Tnmd}^{-/}$mice and their WT littermates were used in this study. The generation of the $\mathrm{Tnmd}^{-1-}$ mice and their primary phenotype were described by Docheva and co-workers. ${ }^{11-13}$ All the mice were on a $\mathrm{C} 57 \mathrm{BL} / 6 \mathrm{~J}$ background. Surgical procedures were performed as previously described by Palmes et al. ${ }^{15}$ with 6 -month old mice that had reached skeletal maturity. In brief, (1) after anesthesia, the skin above the left Achilles tendon was opened from the gastrocnemius muscle to the calcaneus; (2) using sterile scissors, the tendon proper ( $\sim \mathrm{mm}$ above the calcaneus) of the Achilles tendon was fully resected; (3) the tendon ends were then connected with modified Kirchmayr 8-0 Dermalon suture and further supported with single 10-0 Dermalon circular suture; (4) in order to avoid suture failure due to overstretching of the operated tendons, the movement of the talocrural joint was restricted by a cerclage that was inserted through the tibiofibular fork and fixed between the calcaneus and the plantar aponeurosis. This assured a more limited degree of talocrural joint extension $(\sim 30 \%)$ but still allowed a tensile load to be actively transferred to the healing Achilles tendon; and (5) the skin was closed. The tendons were given eight days for repair, corresponding to the early phase of tendon healing, after which the animals were euthanized and the whole hind limb including the Achilles tendongastrocnemius muscle-calcaneus complexes were dissected and histologically processed as described below. All procedures for animal handling prior, during and after surgery were approved by the Animal Care and Use Committee of the Bavarian Government (Grant Nr. 55.2-1-54-2531-57-08). Bio-statistical design of the group size was based on the default values of $\alpha=0.05$ and $\beta=0.8$ for type one error and for the power as well as on pilot histological data for each genotype, resulting in eight animals per group.

Histomorphometry. Achilles tendons within the hind limbs were fixed in $4 \%$ paraformaldehyde (PFA; Merck, Darmstadt, Germany) overnight at $4{ }^{\circ} \mathrm{C}$. After fixation, specimens were decalcified in $10 \%$ ethylene diamine tetraacetic acid (EDTA)/phosphate buffered saline (PBS) pH 8.0 (Sigma-Aldrich, Munich, Germany) for 7 days, and embedded in paraffin or cryogenic media and then sectioned at 5 or 
$10 \mu \mathrm{m}$ for paraffin and frozen specimens, respectively. Every 10th slide was stained with $\mathrm{HE}$ and slides with comparable regional planes between genotypes (where the whole complex of gastrocnemius muscle-Achilles tendon-calcaneus was exposed) were selected for in-depth investigation. To analyze the total histological scores on $\mathrm{HE}$-stained slides we used the established histological scoring system of Stoll et al..$^{17}$ given in Supplementary Table 1. To reveal the ectopic endochondral ossification in the scar tissue, safranin 0 staining was applied using the standard histological protocol.

For immunofluorescence, the tissue sections were treated with $2 \mathrm{mg} / \mathrm{m}$ hyaluronidase (Sigma-Aldrich, Steinheim, Germany) for $30 \mathrm{~min}$ at $37^{\circ} \mathrm{C}$ in order to increase antibody permeability. After washing and blocking with $2 \%$ bovine serum albumin (BSA)/PBS (Sigma-Aldrich), primary antibodies against biglycan, CD68, CD80, CD146, CD163, collagen I, collagen III, collagen IV, Comp, decorin, elastin, fibromodulin, fibronectin, F4/80, lumican, perilipin, p53 and Tnmd (all from Abcam, Cambridge, UK; except for Tnmd, which was provided by Denitsa Docheva) were applied overnight at $4{ }^{\circ} \mathrm{C}$. Next day, corresponding Alexa Fluor 546-labeled secondary antibodies (all from Life technology, Carlsbad, CA, USA) were applied for $1 \mathrm{~h}$. Then, sections were counter-stained with 4',6-diamidino-2-phenylindole (DAPI) (Life Technology) and mounted with fluoroshield (Sigma-Aldrich). To detect proliferating cells, 90 min prior to euthanasia all mice received intraperitoneal injection with BrdU ( $50 \mu \mathrm{g} / \mathrm{g}$ body weight). BrdU detection was performed with a BrdU kit according to the manufacturer's instructions (Roche Applied Science, Penzberg, Germany). To analyze apoptotic cells numbers, TUNEL assay was performed according to the manufacturer's instructions (Abcam). Photo-micrographs were taken on the Observer Z1 microscope equipped with the Axiocam MRm camera (Carl Zeiss, Jena, Germany). In general, all histomorphometry experiments, unless specified otherwise in the text, were reproduced in with 8 animals per group and representative images are shown.

In order to analyze biglycan, Comp and fibronectin levels, an automated quantitative image analysis was performed as described in the literature. ${ }^{18}$ In brief, using ImageJ (National Institutes of Health, Bethesda, MD, USA), the following algorithm was applied: (1) area of interest was manually designated using the 'drawing/selection' tool; (2) 'set measurements for area, integrated density and mean gray value' was selected from the analyze menu; (3) the corrected total cryosection fluorescence (CTCF) representing the biglycan, Comp and fibronectin expression detected were calculated as follows CTCF = media of integrated density - (media of area of selected area $\times$ mean fluorescence). Three animals per group were analyzed.

Scar nuclear density was determined on DAPI staining with ImageJ according to Hsieh and co-workers. ${ }^{18}$ All cell nuclei (DAPI) in 3 images per scar from 3 sections per animal with 8 animals per group were counted. To analyze adipocytes (perilipin), blood vessels (collagen IV), cell proliferation (BrdU), apoptotic cells (TUNEL and p53), TSPC/MSC cells (CD146) and macrophages (CD68, CD80, CD163, and F4/80) quantification of labeled cell per scar tissue was carried out for each staining on 8 animals per group. Each animal was represented with 3 different tissue sections with comparable planes between genotypes. The results were averaged per animal and shown as final mean and standard deviation (S.D.) between the 8 animals per group. The above information is given in the figure legends ( 8 animals per group; each animal represented by 3 tissue sections).

Mouse TSPCs isolation and cell culture. Mouse TSPCs were isolated as previously described by Alberton and co-workers ${ }^{13}$ from tendons of two uninjured $\mathrm{Tnmd}^{-1}$ and WT 6-month-old mice. Tendon tissues were enzymatically treated overnight with collagenase II (Worthington, Lakewood, NJ, USA) in Dulbecco's modified Eagle's medium (DMEM)/Ham's F-12 (1:1) (Biochrom, Berlin, Germany) supplemented with $10 \%$ fetal bovine serum (FBS), $1 \%$ l-ascorbic-acid-2-phosphate (both from Sigma-Aldrich, Steinheim, Germany), 1\% minimum essential medium (MEM)-Amino Acid and 1\% penicillin/streptomycine (Pen/Strep) (both from Biochrom, Berlin, Germany). Then, the cell suspension was filtered through $70 \mu \mathrm{m}$ nylon mesh, centrifuged at $500 \times \mathrm{g}$ for $5 \mathrm{~min}$, and resuspended in fresh culture media. TSPCs were grown at $37{ }^{\circ} \mathrm{C}$ and $5 \% \mathrm{CO}_{2}$ and passaged when $70 \%$ confluent with the culture media changed every third day. Cells in passages 1-3 were used for experiments.

In vitro wound healing assay. These experiments were carried out according to our previously published protocol. ${ }^{59}$ Shortly, $1 \times 10^{4}$ cells per $\mathrm{cm}^{2}$ were plated on collagen I- $(20 \mu \mathrm{g} / \mathrm{Ml}$; Millipore, Billerica, MA, USA) or fibronectin-coated $(10 \mu \mathrm{g} / \mathrm{Ml}$; Sigma-Aldrich, Steinheim, Germany) 6 -well plates in low serum $(2 \%)$ medium and were allowed to form confluent cell layers for $48 \mathrm{~h}$. Prior to imaging, the layers were scratched multiple times. Time lapse photography was performed at
4 frames per $\mathrm{h}$ for $24 \mathrm{~h}$. For each group, the areas of 12 scratches were measured at 9 different time points from 4 independent experiments using the ImageJ 'wound healing' tool.

Migration analysis. Migration analysis was performed similarly to our previous studies. ${ }^{59,60}$ For random migration, $1.5 \times 10^{3} \mathrm{cellls} / \mathrm{cm}^{2}$ of $\mathrm{Tnmd}^{-1-}$ and WT TSPCs were seeded on collagen $\mathrm{I-}(20 \mu \mathrm{g} / \mathrm{ml})$ or fibronectin-coated $(10 \mu \mathrm{g} / \mathrm{ml}) 6$-well plates and incubated for $2 \mathrm{~h}$ before imaging. Time lapse photography was performed at 4 frames per $h$ for $48 \mathrm{~h}$. The image data was extracted with AxioVisionLE software (Carl Zeiss, Jena, Germany) and individual cell tracks were analyzed with ImageJ. Random migration was expressed by calculating the forward migration index (FMl; the ratio of the vector length to the migratory starting point), velocity, and accumulated (cumulative track length) and Euclidian (the ordinary straight-line length between two points) distances. Results of random TSPCs migration measurements consist of 3 independent time lapse movies of two $\mathrm{Tnmd}^{-1-}$ and WT TSPC donors as a total number of 70-80 TSPC per genotype were tracked.

CyQUANT assays. $1.5 \times 10^{3}$ cells per well were plated in 6-well plates, and the CyQUANT assay detection was performed according to the manufacturer's instructions (Invitrogen, Eugene, OR, USA) after 0, 4, 8 and 12 days of cell culture, respectively. CYQUANT assay was repeated independently in 4 experiments per time point with two TSPC donors/genotypes.

Adipogenic differentiation assays. These experiments were carried out according to our previously published protocol. ${ }^{13}$ Briefly, $8 \times 10^{3} \mathrm{cells} / \mathrm{cm}^{2}$ TSPCs were seeded in triplicates in 6-well plates, and were cultivated in an induction media for 5 days (DMEM-high glucose with $10 \% \mathrm{FBS}, 1 \mu \mathrm{M}$ dexamethasone, $200 \mu \mathrm{M}$ indomethacin, $0.01 \mathrm{mg} / \mathrm{ml}$ insulin, and $500 \mu \mathrm{M}$ 3-isobutyl-1-methylxanthine; all from Sigma-Aldrich, Steinheim, Germany) followed by 2 days in preservation media (DMEM-high glucose medium supplemented with $10 \% \mathrm{FBS}, 0.01 \mathrm{mg} / \mathrm{ml}$ insulin). The process was repeated for 21 days. The adipogenic differentiation was estimated by BODIPY 493/503 staining of neutral lipid droplets (Thermo Fisher Scientific, Waltham, MA, USA) and AdipoRed assay (Lonza, Walkersville, MA, USA). Staining was carried out according to the manufacturer's instructions. Using the automatic color pixel quantification tool in the Adobe Photoshop CS5 software, the BODIPY 493/503 staining-positive areas were estimated and calculated as a percentage of the image total pixel size. Using a fluorimeter (Tecan, Männedorf, Switzerland), AdipoRed assays were measured with excitation at $485 \mathrm{~nm}$ and emission at 572 $\mathrm{nm}$. BODIPY 493/503 staining and AdipoRed assay were repeated in 4 independent experiments.

ELISA. The protein levels of biglycan and fibronectin were analyzed by ELISA. TSPCs $\left(8 \times 10^{3}\right.$ cells $\left./ \mathrm{cm}^{2}\right)$ were seeded in 6 -well plates. After 3 days, the cell supernatant and cell RIPA protein lysates were collected and frozen. Before ELISA, the total protein of all samples was measured via DC protein assays (BioRad, Munich, Germany). Secreted biglycan and fibronectin were determined using mouse biglycan and fibronectin ELISA kits (Cloud-Clone Corp, Katy, TX, USA; and Aviva Systems Biology, San Diego, CA, USA; respectively) according to the manufacturer's instructions. Two independent ELISA measurements were done with two donors/genotypes. The data was expressed as target-specific concentration to total protein content.

Semi-quantitative and qRT-PCR. Total RNA from tendon tissue and adipogenic-stimulated TSPCs was isolated with Qiagen RNeasy Mini kit (Qiagen, Hilden, Germany) and used for semi-quantitative and qRT-PCR. For cDNA synthesis, $1 \mu \mathrm{g}$ total RNA and AMV First-Strand cDNA Synthesis Kit (Invitrogen) were used. Semi-quantitative PCR was performed with Taq DNA Polymerase (Invitrogen) in MGResearch instrument (BioRad, Munich, Germany). For Primer sequences and PCR conditions: Ppary forward 5'ctccgtgatggaagaccactc $3^{\prime}$, reverse 5'agactcggaactcaatggc3'; Lpl forward 5' $5^{\prime}$ tctggctgacactggacaa3', reverse $5^{\prime}$ tgggccattagattcctcac3'; Fabp4 forward 5'gaagcttgtctccagtcaaaa3', reverse 5' agtcacgccttcataacacat3'; Gapdh forward 5'gagaggccctatcccaactc3', reverse 5' gtgggtgcagcgaacttat3'; PCR was performed with incubation at $94^{\circ} \mathrm{C}$ for 5 min followed by 30 cycles of a three step temperature program of $1 \mathrm{~min}$ at $94^{\circ} \mathrm{C}, 20 \mathrm{~s}$ at $60^{\circ} \mathrm{C}$, and $30 \mathrm{~s}$ at $72{ }^{\circ} \mathrm{C}$. The PCR reaction was terminated after a 7 min extension at $70^{\circ} \mathrm{C}$. The band intensity of the amplified products in the gel was visualized, photographed and analyzed using a gel imager (Vilber Lourmat, Eberhardzell, Germany). The relative gene expression was quantified by densitometry and normalized to the amount of Gapdh with ImageJ and presented as fold-change to 
WT. Quantitative RT-PCR of adipogenic and tenogenic associated gene markers was performed using RealTime Ready Custom Panel 96-32+ plates (https:// configurator.realtimeready.roche.com) according to the manufacturer's instructions (Roche Applied Science, Mannheim, Germany). Briefly, PCR reactions were pipetted on ice and each well contained $10 \mu \mathrm{l}$ LightCycler 480 probes master mix, $0.2 \mu \mathrm{l} \mathrm{CDNA}$ and $9.8 \mu \mathrm{l} \mathrm{PCR}$ grade water. Plates were subsequently sealed and centrifuged down for $15 \mathrm{~s}$ at $2100 \mathrm{rpm}$. The relative gene expression was calculated as describe by Dex and co-workers. ${ }^{12}$ All PCR results have been reproduced independently in five experiments.

Statistical analysis. Statistical differences between two groups were determined using two-tailed unpaired Student's $t$-test, or two-tailed non-parametric Mann-Whitney test. Sample size and experimental reproduction are indicated for each method. Results are presented as mean \pm S.D. Differences were considered statistically significant according to values of ${ }^{\star} P<0.05,{ }^{* *} P<0.01$ and ${ }^{* * *} P<0.001$.

\section{Conflict of Interest}

The authors declare no conflict of interest.

Acknowledgements. DD acknowledges the German Research Foundation (Grant No. D01414/3-1) and the National Institutes of Health (Grant No. GM089820). DL acknowledges the National Natural Science Foundation of China (Grant No. 81600696). We are thankful to CF Hsieh, S Dex and D Drenkard for experimental help. We also thank Dr G Pattappa and Professor B Johnstone for proof-reading of the manuscript.

\section{Author contributions}

$\mathrm{DL}$ designed, performed and analyzed experiments and wrote the manuscript; PA assisted with the operations, performed tissue dissections and analyses; MDC performed ELISA and semi-quantitative RT-PCR; EV performed tendon surgery; MS approved manuscript; DD conceived the study, designed, and analyzed experiments and wrote the manuscript.

\section{Publisher's Note}

Springer Nature remains neutral with regard to jurisdictional claims in published maps and institutional affiliations.

1. Nourissat G, Berenbaum F, Duprez D. Tendon injury: from biology to tendon repair. Nat Rev Rheumatol 2015; 11: 223-233.

2. Galatz LM, Gerstenfeld L, Heber-Katz E, Rodeo SA. Tendon regeneration and scar formation: the concept of scarless healing. J Orthop Res 2015; 33: 823-831.

3. Docheva D, Müller SA, Majewski M, Evans CH. Biologics for tendon repair. Adv Drug Deliv Rev 2015; 84: 222-239.

4. Gaspar D, Spanoudes K, Holladay C, Pandit A, Zeugolis D. Progress in cell-based therapies for tendon repair. Adv Drug Deliv Rev 2015; 84: 240-256.

5. Aslan H, Kimelman-Bleich N, Pelled G, Gazit D. Molecular targets for tendon neoformation. $J$ Clin Invest 2008; 118: 439-444.

6. Brandau O, Meindl A, Fässler R, Aszódi A. A novel gene, tendin, is strongly expressed in tendons and ligaments and shows high homology with chondromodulin-I. Dev Dyn 2001; 221: $72-80$

7. Shukunami C, Oshima Y, Hiraki Y. Molecular cloning of tenomodulin, a novel chondromodulin-I related gene. Biochem Biophys Res Commun 2001; 280: 1323-1327.

8. Yamana K, Wada H, Takahashi Y, Sato H, Kasahara Y, Kiyoki M. Molecular cloning and characterization of CHM1L, a novel membrane molecule similar to chondromodulin-I. Biochem Biophys Res Commun 2001; 280: 1101-1106.

9. Dex S, Lin D, Shukunami C, Docheva D. Tenogenic modulating insider factor: Systematic assessment on the functions of tenomodulin gene. Gene 2016; 587: 1-17.

10. Oshima Y, Sato K, Tashiro F, Miyazaki J, Nishida K, Hiraki Y et al. Anti-angiogenic action of the C-terminal domain of tenomodulin that shares homology with chondromodulin-l. J Cell Sci 2004; 117: 2731-2744.

11. Docheva D, Hunziker EB, Fässler R, Brandau O. Tenomodulin is necessary for tenocyte proliferation and tendon maturation. Mol Cell Biol 2005; 25: 699-705.

12. Dex S, Alberton P, Willkomm L, Söllradl T, Bago S, Milz $S$ et al. Tenomodulin is required for tendon endurance running and collagen I fibril adaptation to mechanical load. EBioMedicine 2017; 20: 240-254

13. Alberton P, Dex S, Popov C, Shukunami C, Schieker M, Docheva D. Loss of tenomodulin results in reduced self-renewal and augmented senescence of tendon stem/progenitor cells. Stem Cells Dev 2015; 24: 597-609.
14. Jiang Y, Shi Y, He J, Zhang Z, Zhou G, Zhang W et al. Enhanced tenogenic differentiation and tendon-like tissue formation by tenomodulin overexpression in murine mesenchymal stem cells. J Tissue Eng Regen Med 2016; 11: 2525-2536.

15. Palmes D, Spiegel HU, Schneider TO, Langer M, Stratmann U, Budny T et al. Achilles tendon healing: long-term biomechanical effects of postoperative mobilization and immobilization in a new mouse model. J Orthop Res 2002; 20: 939-946.

16. Dyment NA, Hagiwara Y, Matthews BG, Li Y, Kalajzic I, Rowe DW. Lineage tracing of resident tendon progenitor cells during growth and natural healing. PLOS One 2014; 9 : e96113.

17. Stoll C, John T, Conrad C, Lohan A, Hondke S, Ertel W et al. Healing parameters in a rabbit partial tendon defect following tenocyte/biomaterial implantation. Biomaterials 2011; 32 : 4806-4815.

18. Hsieh CF, Alberton P, Loffredo-Verde E, Volkmer E, Pietschmann M, Müller PE et al. Periodontal ligament cells as alternative source for cell-based therapy of tendon injuries: in vivo study of full-size Achilles tendon defect in a rat model. Eur Cell Mater 2016; 32 228-240.

19. Fang Z, Zhu T, Shen WL, Tang QM, Chen JL, Yin Z et al. Transplantation of fetal instead of adult fibroblasts reduces the probability of ectopic ossification during tendon repair. Tissue Eng Part A 2014; 20: 1815-1826.

20. Lui PP, Cheuk YC, Lee YW, Chan KM. Ectopic chondro-ossification and erroneous extracellular matrix deposition in a tendon window injury model. J Orthop Res 2012; 30 : 37-46.

21. Poulsen RC, Knowles HJ, Carr AJ, Hulley PA. Cell differentiation versus cell death: extracellular glucose is a key determinant of cell fate following oxidative stress exposure. Cell Death Dis 2014; 5: e1074.

22. Bi Y, Ehirchiou D, Kilts TM, Inkson CA, Embree MC, Sonoyama $W$ et al. Identification of tendon stem/progenitor cells and the role of the extracellular matrix in their niche. Nat Med 2007; 13: 1219-1227.

23. Lee $\mathrm{CH}$, Lee FY, Tarafder S, Kao K, Jun Y, Yang G et al. Harnessing endogenous stem/ progenitor cells for tendon regeneration. J Clin Invest 2015; 125: 2690-2701.

24. Yin Z, Hu JJ, Yang L, Zheng ZF, An CR, Wu BB et al. Single-cell analysis reveals a nestin+ tendon stem/progenitor cell population with strong tenogenic potentiality. Sci Adv 2016; 2 . e1600874.

25. Chazaud B. Macrophages: supportive cells for tissue repair and regeneration. Immunobiology 2014; 219: 172-178.

26. Thomopoulos S, Parks WC, Rifkin DB, Derwin KA. Mechanisms of tendon injury and repair J Orthop Res 2015; 33: 832-839.

27. Shukunami C, Yoshimoto Y, Takimoto A, Yamashita H, Hiraki Y. Molecular characterization and function of tenomodulin, a marker of tendons and ligaments that integrate musculoskeletal components. Jpn Dent Sci Rev 2016; 52: 84-92.

28. Wu YF, Chen $\mathrm{CH}$, Cao Y, Avanessian B, Wang XT, Tang JB. Molecular events of cellular apoptosis and proliferation in the early tendon healing period. J Hand Surg Am 2010; 35: 2-10.

29. Tokunaga T, Shukunami C, Okamoto N, Taniwaki T, Oka K, Sakamoto H et al. FGF-2 stimulates the growth of tenogenic progenitor cells to facilitate the generation of Tenomodulin-positive tenocytes in a rat rotator cuff healing model. Am J Sports Med 2015; 43: 2411-2422.

30. Kang JR, Gupta R. Mechanisms of fatty degeneration in massive rotator cuff tears J Shoulder Elbow Surg 2012; 21: 175-180.

31. Oak NR, Gumucio JP, Flood MD, Saripalli AL, Davis ME, Harning JA et al. Inhibition of 5LOX, COX-1, and COX-2 increases tendon healing and reduces muscle fibrosis and lipid accumulation after rotator cuff repair. Am J Sports Med 2014; 42: 2860-2868.

32. Gladstone JN, Bishop JY, Lo IK, Flatow EL. Fatty infiltration and atrophy of the rotator cuff do not improve after rotator cuff repair and correlate with poor functional outcome. Am J Sports Med 2007; 35: 719-728

33. Zhou Z, Akinbiyi T, Xu L, Ramcharan M, Leong DJ, Ros SJ et al. Tendon-derived stem/ progenitor cell aging: defective self-renewal and altered fate. Aging Cell 2010; 9: 911-915.

34. Kannus $\mathrm{P}$, Józsa $\mathrm{L}$. Histopathological changes preceding spontaneous rupture of a tendon. A controlled study of 891 patients. J Bone Joint Surg Am 1991; 73: 1507-1525.

35. Nanthakumar CB, Hatley RJ, Lemma S, Gauldie J, Marshall RP, Macdonald SJ. Dissecting fibrosis: therapeutic insights from the small-molecule toolbox. Nat Rev Drug Discov2015; 14 693-720.

36. Pellicoro A, Ramachandran P, Iredale JP, Fallowfield JA. Liver fibrosis and repair: immune regulation of wound healing in a solid organ. Nat Rev Immunol 2014; 14: 181-194.

37. Wynn TA, Ramalingam TR. Mechanisms of fibrosis: therapeutic translation for fibrotic disease. Nat Med 2012; 18: 1028-1040.

38. Chen S, Jiang S, Zheng W, Tu B, Liu S, Ruan H et al. RelA/p65 inhibition prevents tendon adhesion by modulating inflammation, cell proliferation, and apoptosis. Cell Death Dis 2017; 8: e2710.

39. Shen H, Kormpakis I, Havlioglu N, Linderman SW, Sakiyama-Elbert SE, Erickson IE et al. The effect of mesenchymal stromal cell sheets on the inflammatory stage of flexor tendon healing. Stem Cell Res Ther 2016; 7: 144.

40. Manning CN, Havlioglu N, Knutsen E, Sakiyama-Elbert SE, Silva MJ, Thomopoulos S et al. The early inflammatory response after flexor tendon healing: a gene expression and histological analysis. J Orthop Res 2014; 32: 645-652.

41. Geary MB, Orner CA, Bawany F, Awad HA, Hammert WC, O'Keefe RJ et al. Systemic EP4 inhibition increases adhesion formation in a murine model of flexor tendon repair. PLOS One 2015; 10: e0136351. 
42. Beredjiklian PK. Biologic aspects of flexor tendon laceration and repair. J Bone Joint Surg Am 2003; 85-A: 539-550.

43. Korntner S, Kunkel N, Lehner C, Gehwolf R, Wagner A, Augat P et al. A high-glucose diet affects Achilles tendon healing in rats. Sci Rep 2017; 7: 780 .

44. Spiegelman BM, Ginty CA. Fibronectin modulation of cell shape and lipogenic gene expression in 3T3-adipocytes. Cell 1983; 35: 657-666.

45. Gelberman RH, Linderman SW, Jayaram R, Dikina AD, Sakiyama-Elbert S, Alsberg E et al. Combined administration of ASCs and BMP-12 promotes an M2 macrophage phenotype and enhances tendon healing. Clin Orthop Relat Res 475: 2318-23312017.

46. Senol-Cosar O, Flach RJ, DiStefano M, Chawla A, Nicoloro S, Straubhaar J et al. Tenomodulin promotes human adipocyte differentiation and beneficial visceral adipose tissue expansion. Nat Commun 2016; 7: 10686.

47. Tolppanen AM, Pulkkinen L, Herder C, Koenig W, Kolehmainen M, Lindström J et al. The genetic variation of the tenomodulin gene (TNMD) is associated with serum levels of systemic immune mediators-the Finnish Diabetes Prevention Study. Genet Med 2008; 10: 536-544.

48. Saiki A, Olsson M, Jernås M, Gummesson A, McTernan PG, Andersson J et al. Tenomodulin is highly expressed in adipose tissue, increased in obesity, and down-regulated during dietinduced weight loss. J Clin Endocrinol Metab 2009; 94: 3987-3994.

49. Tolppanen AM, Kolehmainen M, Pulkkinen L, Uusitupa M. Tenomodulin gene and obesityrelated phenotypes. Ann Med 2010; 42: 265-275.

50. Tolppanen AM, Pulkkinen L, Kuulasmaa T, Kolehmainen M, Schwab U, Lindström J et al. The genetic variation in the tenomodulin gene is associated with serum total and LDL cholesterol in a body size-dependent manner. Int J Obes 2008; 32: 1868-1872.

51. Johansson LE, Danielsson AP, Parikh H, Klintenberg M, Norström F, Groop L et al. Differential gene expression in adipose tissue from obese human subjects during weight loss and weight maintenance. Am J Clin Nutr 2012; 96: 196-207.

52. Kolehmainen M, Salopuro T, Schwab US, Kekäläinen J, Kallio $P$, Laaksonen DE et al. Weight reduction modulates expression of genes involved in extracellular matrix and cell death: the GENOBIN study. Int J Obes 2008; 32: 292-303.

53. Kawai M, Rosen CJ. PPAR $\gamma$ : a circadian transcription factor in adipogenesis and osteogenesis. Nat Rev Endocrinol 2010; 6: 629-636.

54. Ahmadian M, Suh JM, Hah N, Liddle C, Atkins AR, Downes M et al. PPAR $\gamma$ signaling and metabolism: the good, the bad and the future. Nat Med 2013; 19: 557-566.
55. Cao Y, Gomes SA, Rangel EB, Paulino EC, Fonseca TL, Li J et al. S-nitrosoglutathione reductase-dependent PPAR $\gamma$ denitrosylation participates in MSC-derived adipogenesis and osteogenesis. J Clin Invest 2015; 125: 1679-1691.

56. Rosen ED, MacDougald OA. Adipocyte differentiation from the inside out. Nat Rev Mol Cell Biol 2006; 7: 885-896.

57. Gehwolf R, Wagner A, Lehner C, Bradshaw AD, Scharler C, Niestrawska JA et al. Pleiotropic roles of the matricellular protein Sparc in tendon maturation and ageing. Sci Rep 2016; 6: 32635 .

58. Nobusue H, Onishi N, Shimizu T, Sugihara E, Oki Y, Sumikawa $Y$ et al. Regulation of MKL1 via actin cytoskeleton dynamics drives adipocyte differentiation. Nat Commun 2014; 5: 3368.

59. Kohler J, Popov C, Klotz B, Alberton P, Prall WC, Haasters F et al. Uncovering the cellular and molecular changes in tendon stem/progenitor cells attributed to tendon aging and degeneration. Aging Cell 2013; 12: 988-999.

60. Popov C, Kohler J, Docheva D. Activation of EphA4 and EphB2 reverse signaling restores the age-associated reduction of self-renewal, migration, and actin turnover in human tendon stem/progenitor cells. Front Aging Neurosci 2016; 7: 246.

cublished by Nature Publishing Group. This work is

licensed under a Creative Commons Attribution 4.0 International License. The images or other third party material in this article are included in the article's Creative Commons license, unless indicated otherwise in the credit line; if the material is not included under the Creative Commons license, users will need to obtain permission from the license holder to reproduce the material. To view a copy of this license, visit http://creativecommons.org/licenses/by/4.0/

(C) The Author(s) 2017

Supplementary Information accompanies this paper on Cell Death and Disease website (http://www.nature.com/cddis) 